\title{
REVIEW ARTICLE Three-dimensional Ultrasound in Infertility and Ectopic Pregnancy
}

\author{
Sonal Panchal ${ }^{1}$, Chaitanya Nagori ${ }^{2}$
}

\begin{abstract}
Three-dimensional ultrasound (3D US) is now a well-developed technology; and it has proved its superiority over the B mode US in several applications. Infertility may be due to female factor as well as male factor (40-45\%). For the assessment of the female, transvaginal scan is the scanning method of choice and the first-line investigation. Adding dynamic examination, Doppler and 3D US markedly increases the information available. Three-dimensional US can be used for assessment of uterine congenital anomalies, intrauterine pathologies, adnexal lesions, tubal patency assessment, polycystic ovaries, ovarian follicular monitoring, and endometrial receptivity assessment. Three-dimensional US can better demonstrate the uterine lesions, such as polyps, endometrial synechiae, and fibroids. Better delineation of endometriomyometrial junction helps diagnosis of adenomyosis comparable to MRI. Tubal assessment can be made more effective by adding 3D to hysterosalpingocontrast sonography. Three-dimensional US is accurate for volume assessment for both follicles and endometrium, which are much more reliable parameters than follicular diameter or endometrial thickness. The presence of cumulus, the presence of which can be confirmed by 3D US more easily than with B mode and increases the surety of the presence of a mature ova in the follicle. Three-dimensional power Doppler gives an idea about global vascularity of the follicle and endometrium. However, larger studies are still needed to establish more precise values of 3D ultrasonography in the assessment of female infertility.
\end{abstract}

Keywords: Ectopic pregnancy, Female infertility, Three-dimensional ultrasonography, Uterine congenital malformations.

Donald School Journal of Ultrasound in Obstetrics and Gynecology (2019): 10.5005/jp-journals-10009-1609

\section{INTRODUCTION}

Three-dimensional ultrasound is now a well-developed technology, and it has proved its superiority over the B mode ultrasound (US) in several applications. In patients with infertility or gynecological complaints, some abnormalities are suspected in the uterus, tubes, or ovaries in the female patients, but the male factor is also equally important in infertility. For the assessment of the female, transvaginal scan is the scanning method of choice and the first-line investigation. Adding dynamic examination, Doppler and 3D US markedly increase the information available. Threedimensional US can be used for assessment of uterine congenital anomalies, intrauterine pathologies, adnexal lesions, tubal patency assessment, polycystic ovaries, ovarian follicular monitoring, and endometrial receptivity assessment. In infertile male patients, $3 D$ US has a role in the assessment of the seminal vesicles and ejaculatory ducts, especially with transrectal US. This means that it may help differentiate between obstructive and non-obstructive azoospermia. Though in this article, we discuss the role of 3D US in female infertility.

\section{D ultrasound for Abnormalities of the UTERUS}

Amongst the uterine abnormalities, mullerian abnormalities and fibroids have been discussed elsewhere in this book. So in this article, we discuss the other abnormalities of the uterus that affect fertility. These include endometritis, polyps, and adenomyosis, chiefly. Though endometritis is a B mode and Doppler diagnosis, polyps, and adenomyosis have a definite role in 3D US.

\section{Endometrial Polyp}

Polyp is a soft tissue solid projection from the endometrial wall into the endometrial cavity. This may have a pedicle or may be sessile. Polyps may be of variable sizes and may involve the endometrial
1,2Dr Nagori's Institute for Infertility and IVF, Ahmedabad, Gujarat, India Corresponding Author: Sonal Panchal, Dr Nagori's Institute for Infertility and IVF, Ahmedabad, Gujarat, India, Phone: +91 9824050911 , e-mail: sonalyogesh@yahoo.com

How to cite this article: Panchal S, Nagori C. Three-dimensional Ultrasound in Infertility and Ectopic Pregnancy. Donald School J Ultrasound Obstet Gynecol 2019;13(4):193-215.

Source of support: Nil

Conflict of interest: None

cavity and may also arise in the cervical canal. Polyps are fairly easy to diagnose during peri-ovulatory phase of the menstrual cycle (Fig. 1). However, polyps are obscured in the secretory phase due

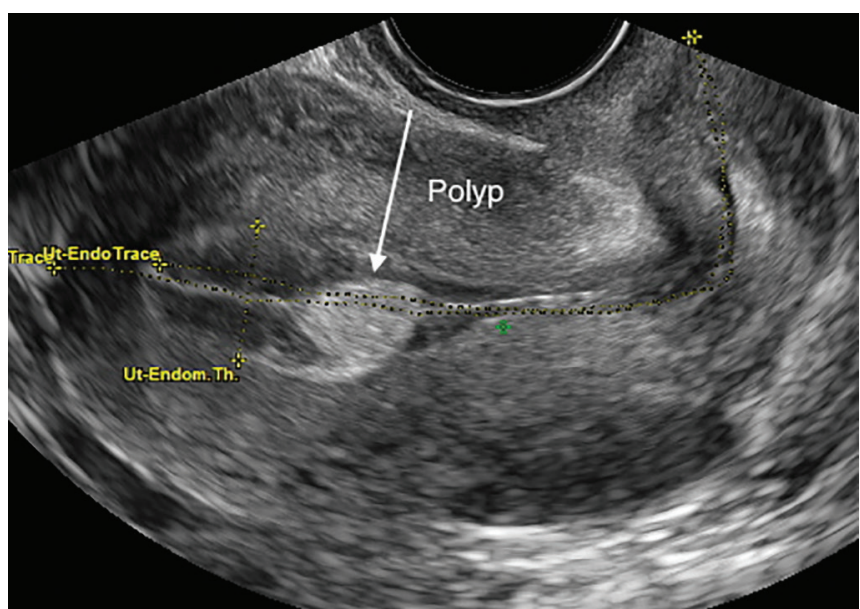

Fig. 1: B mode ultrasound image showing endometrial polyp in preovulatory phase 


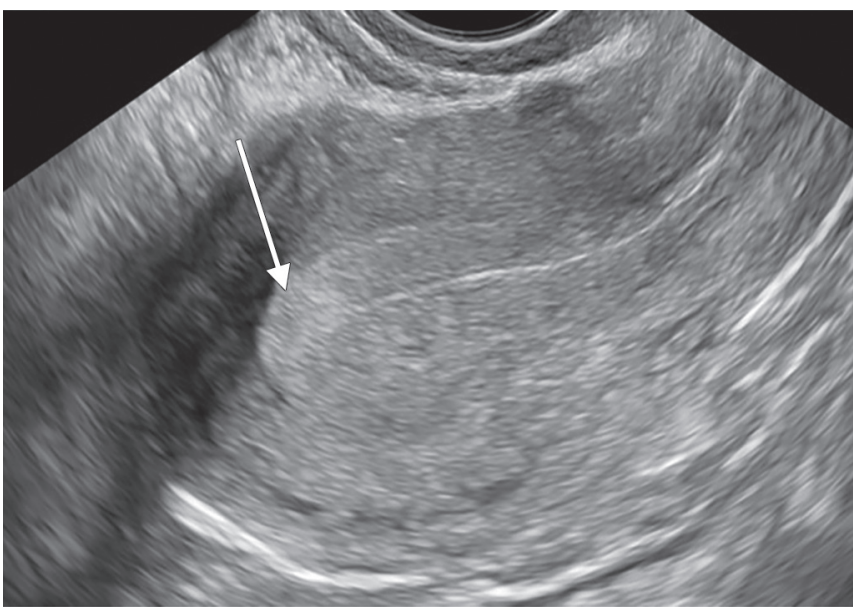

Fig. 2: B mode ultrasound image showing endometrial polyp in secretory phase

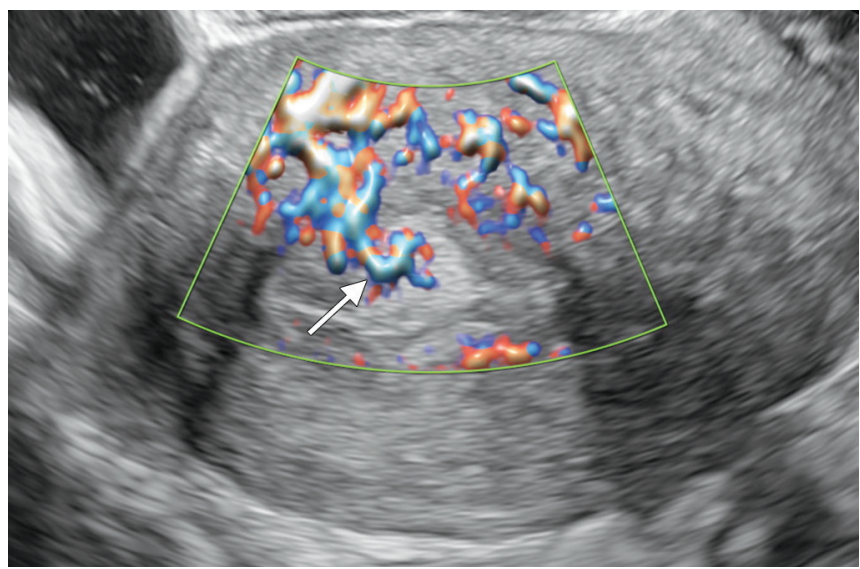

Fig. 3: High definition flow showing single feeding vessel in the polyp
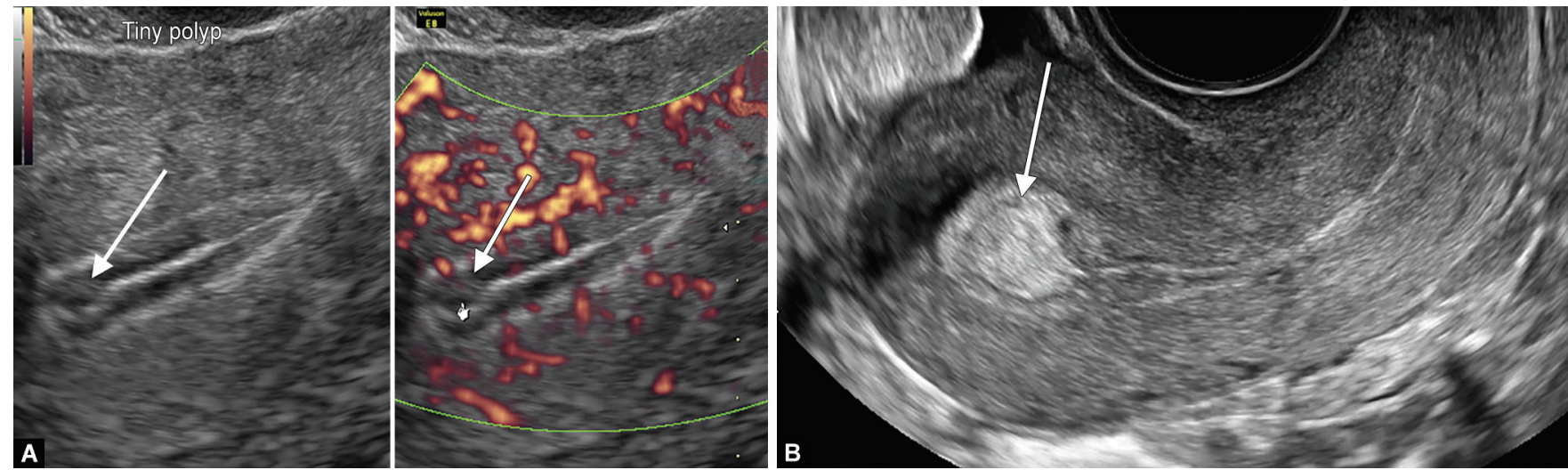

Figs 4A and B: (A) B mode and power Doppler image showing a small polyp; (B) B mode ultrasound image of a polyp filling the fundal endometrium

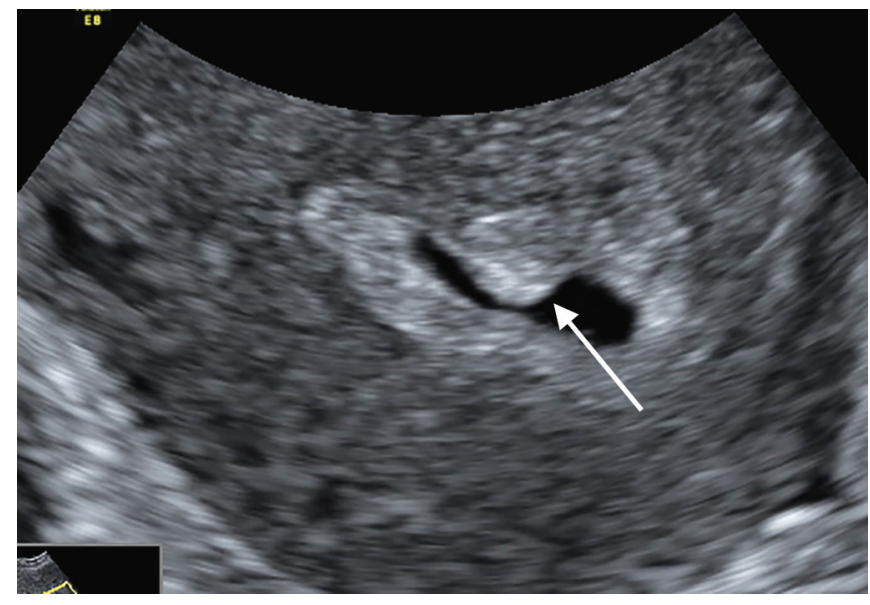

Fig. 5: B mode ultrasound image of a small polyp on sonohysterography

to the hyperechoic endometrium. In such cases, localized area of subtle increased echogenicity (Fig. 2), with Doppler showing a single feeding vessel may be diagnostic. Single feeding vessel has high sensitivity and specificity for the diagnosis of polyps ${ }^{1}$ (Fig. 3).

In early proliferative phase when the endometrium is thin, smaller polyps can be easily missed (Fig. 4) but the larger ones may show bulbous irregularity of the endometrium. Threedimensional US helps differentiate large polyps with sessile base from hyperplasia. Whatever the phase of the cycle, saline infusion sonohysterography is the investigation, that will allow the lesion to be clearly visualized (Fig. 5). But 3D US with sonohysterography is the mostly reliable US modality for diagnosis of polyp.

\begin{tabular}{ll}
\hline Sensitivity & $81.2 \%$ \\
Specificity & $88.2 \%$ \\
Positive-predictive values & $92.9 \%$ \\
Negative-predictive values & $71.4 \%$ \\
\hline
\end{tabular}

\section{Sonohysterography Technique}

A six French Foley's catheter with external diameter of $1.6 \mathrm{~mm}$ and an internal diameter of $1.1 \mathrm{~mm}$ is introduced through the cervix and was placed so that the bulb remains in the cervix. Distend the balloon by $1.5-2 \mathrm{~mL}$ of distilled water. Slowly inject 5-10 mL of normal saline with $20 \mathrm{~mL}$ syringe. Introduce the probe into the vagina after removing the speculum and tenaculum, but with a catheter in situ. While filling the syringe and injecting the fluid, take care not to introduce any air, which will cause artifacts. Take a 3D sweep of the uterus and reconstruct in coronal view. Twodimensional US may also be used with fluid in situ for identifying endometrial pathologies. Lately, gel has been used instead of saline and has been found to be more convenient with equivalent results. Normal saline gives negative contrast better for intracavitary lesions. Instillagel and endosgel are the commonly available preparations. These contain lidocaine hydrochloride, $20 \mathrm{mg} / \mathrm{g}$, chlorhexidine 

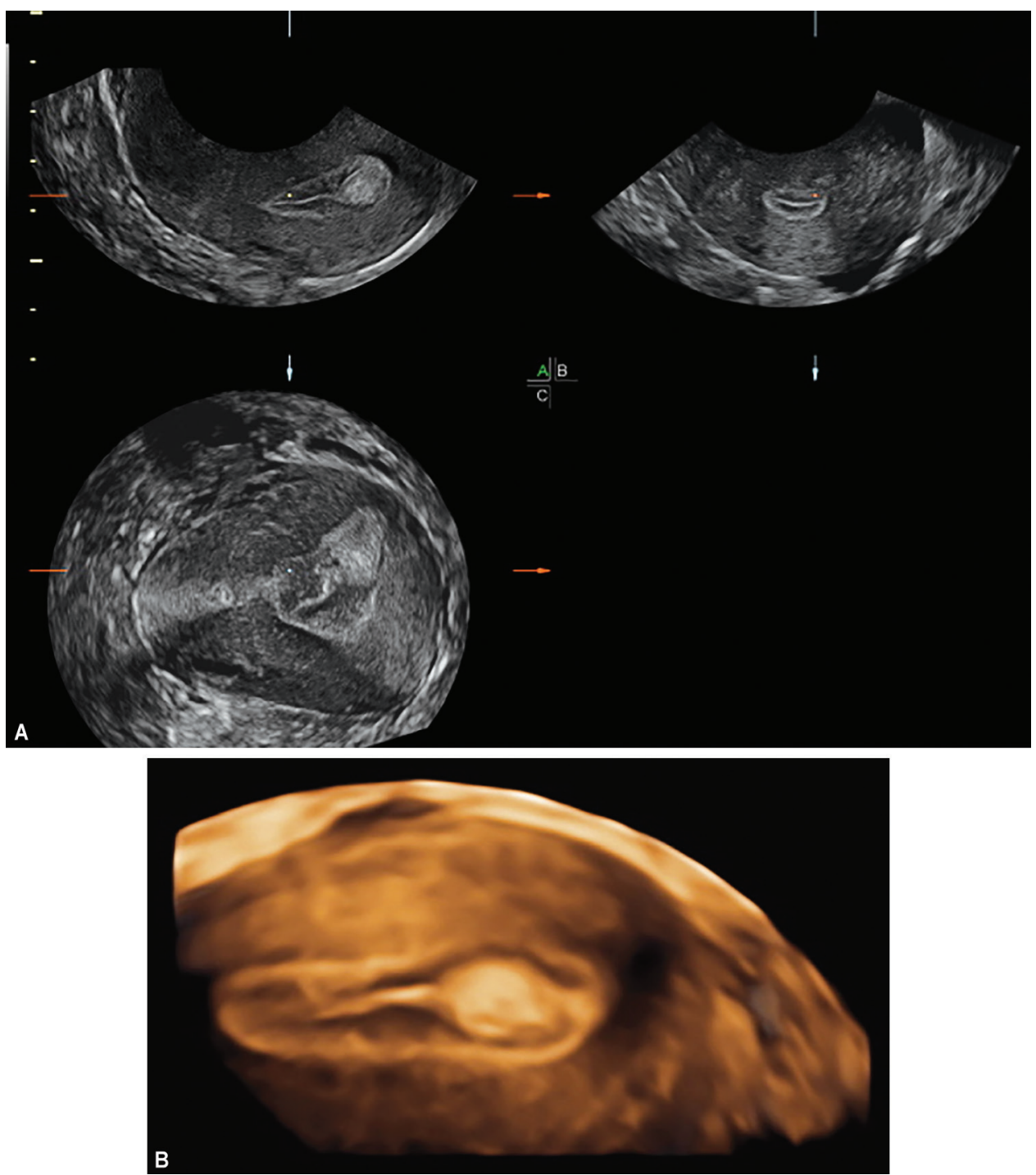

Figs 6A and B: (A) 3D ultrasound multiplanar image of the endometrial polyp; (B) Polyp seen with clear margins and high contrast on 4D ultrasound volume contrast imaging $A$

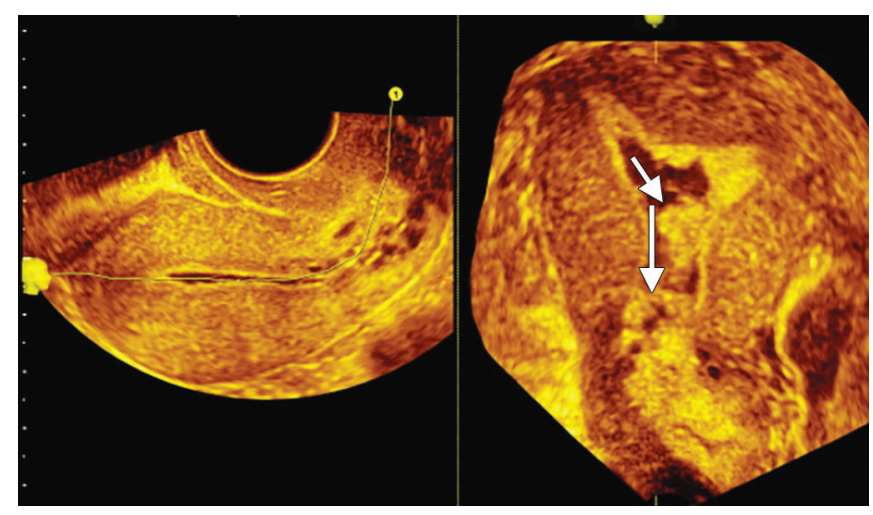

Fig. 7: 3D ultrasound, volume contrast imaging $C$ image of the uterus showing multiple polyps in coronal section

digluconate, methyl hydroxybenzoate, propyl hydroxybenzoate, sodium lactate, hydroxyethylcellulose, and purified water. It does not flow out the uterus very quickly because of its viscosity.

Three-dimensional US techniques used for diagnosis and demonstration of polyps are as follows:
Volume contrast imaging ( $V C I) A$ : This is a thick slice imaging and therefore increases the contrast of the subtle lesions. This significantly improves the visualization of small polyps. Depending on the size of the polyp, the slice thickness of the $\mathrm{VCl}$ is selected (Fig. 6). Volume contrast imaging can also be used to see the coronal plane, and this is called $\mathrm{VCl}$ C. This gives rendered like images (Fig. 7).

Three-dimensional rendering: Three-dimensional volume acquired with or without sonohysterography and rendering will demonstrate the polyp. Rendering can also demonstrate the exact location of the polyp in the endometrial cavity, the pedicle location, and the thickness of the pedicle, which can be of help to plan the surgery (Fig. 8).

\section{Endometrial Hyperplasia}

Overgrowth of endometrial epithelium-endometrial hyperplasia leads to partial or generalized thickening of the endometrium with a broad base on the endometrium, unlike the polyp. On $3 \mathrm{D}$, it is seen as a thickening of one or both lips, complete or incomplete. The endometromyometrial junction-hypoechoic halo is well preserved (Fig. 9). Doppler shows multiple vessels 


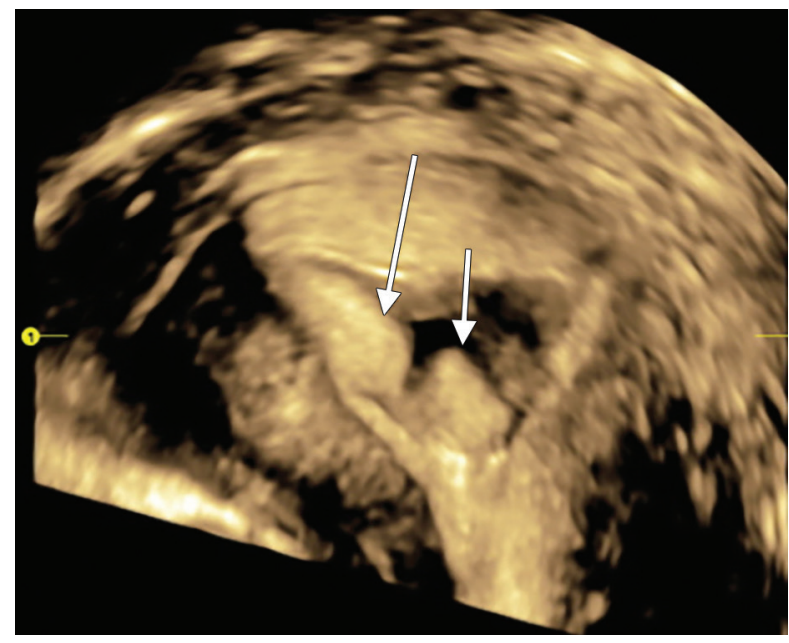

Fig. 8: 3D ultrasound rendered image of sonohysterography showing two polyps (arrows)

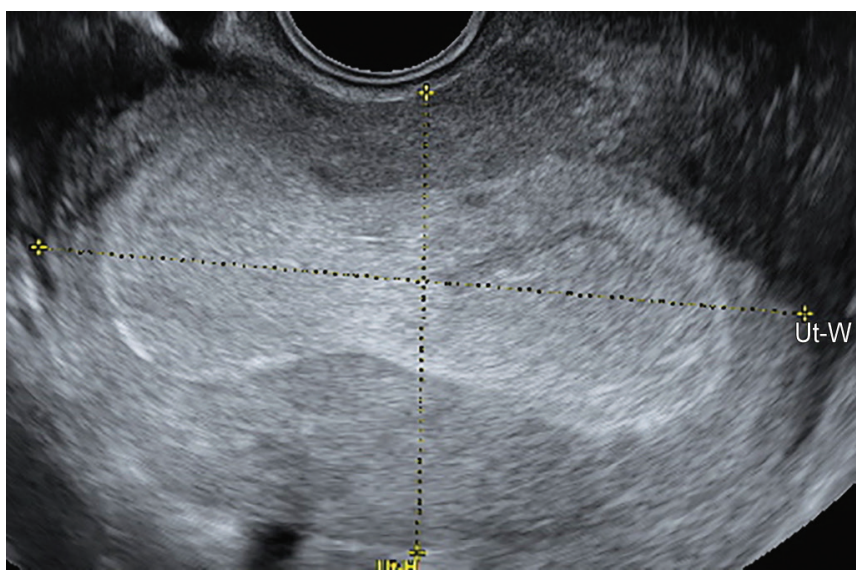

Fig. 9: B mode ultrasound image of the uterus showing endometrial hyperplasia
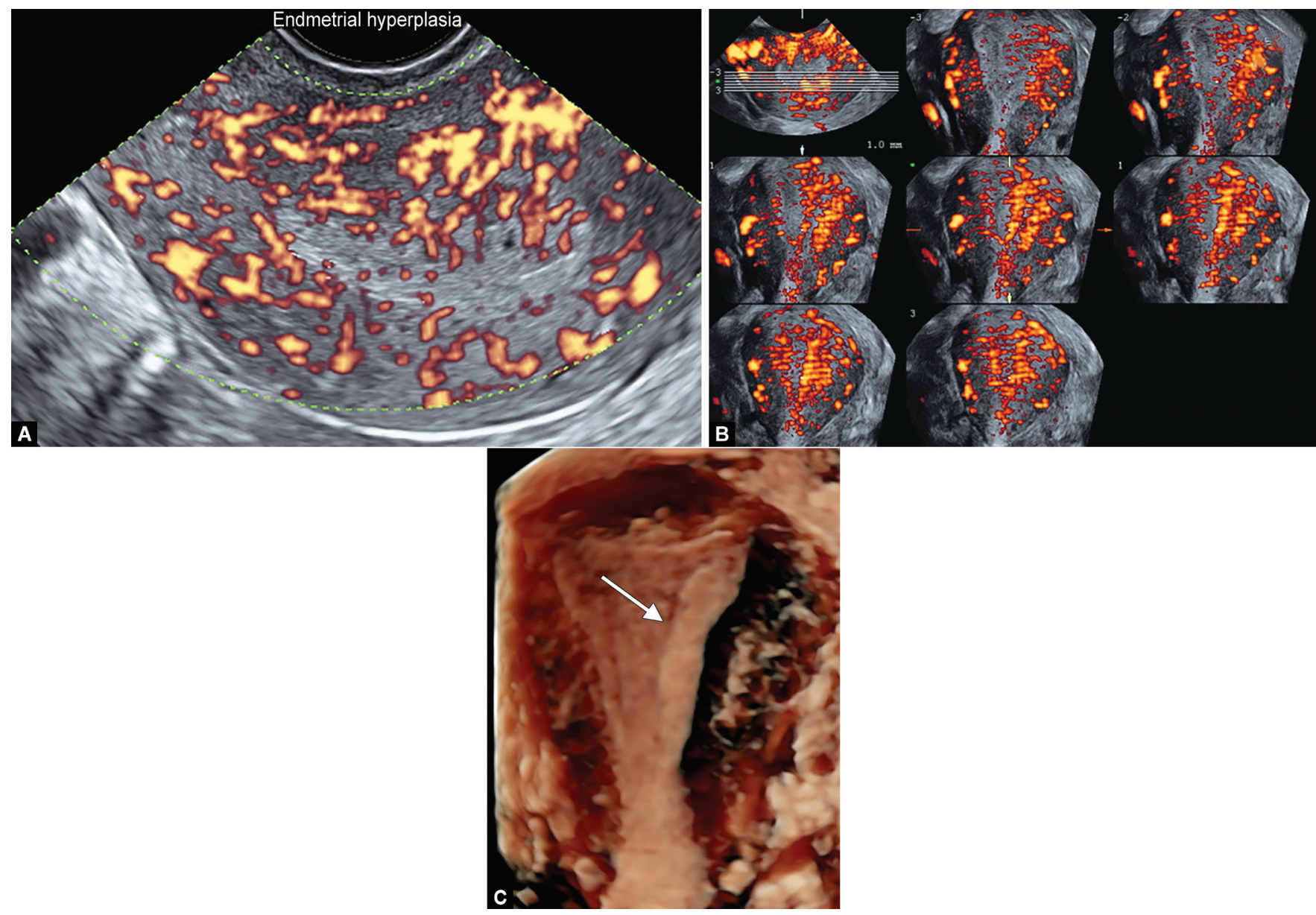

Figs 10A to C: (A) Power Doppler image of endometrial hyperplasia showing symmetrical distribution of blood vessels; (B) 3D power Doppler image showing endometrial hyperplasia and symmetrically distributed blood vessels; (C) 3D rendered image of endometrial hyperplasia

symmetrically arranged, supplying this thickening, which are regularly placed and can be better confirmed on 3D power Doppler (Fig. 10).

\section{Intrauterine Device Placement}

Intrauterine device (IUD) is properly placed if the distance between the fundus and tip of the IUD $<5 \mathrm{~mm}$ on rendered coronal image or distance from tip of IUD to outer wall of the uterine fundus $\leq$ (ant. wall + post. wall) $/ 2 \times 1.33$. For displaced IUD, US is the choice of investigation. It not only demonstrates the exact location of IUD but also determines the route of removal whether abdominal or hysteroscopic. Three-dimensional US is the modality of choice to assess the displacement of IUD (Fig. 11). 

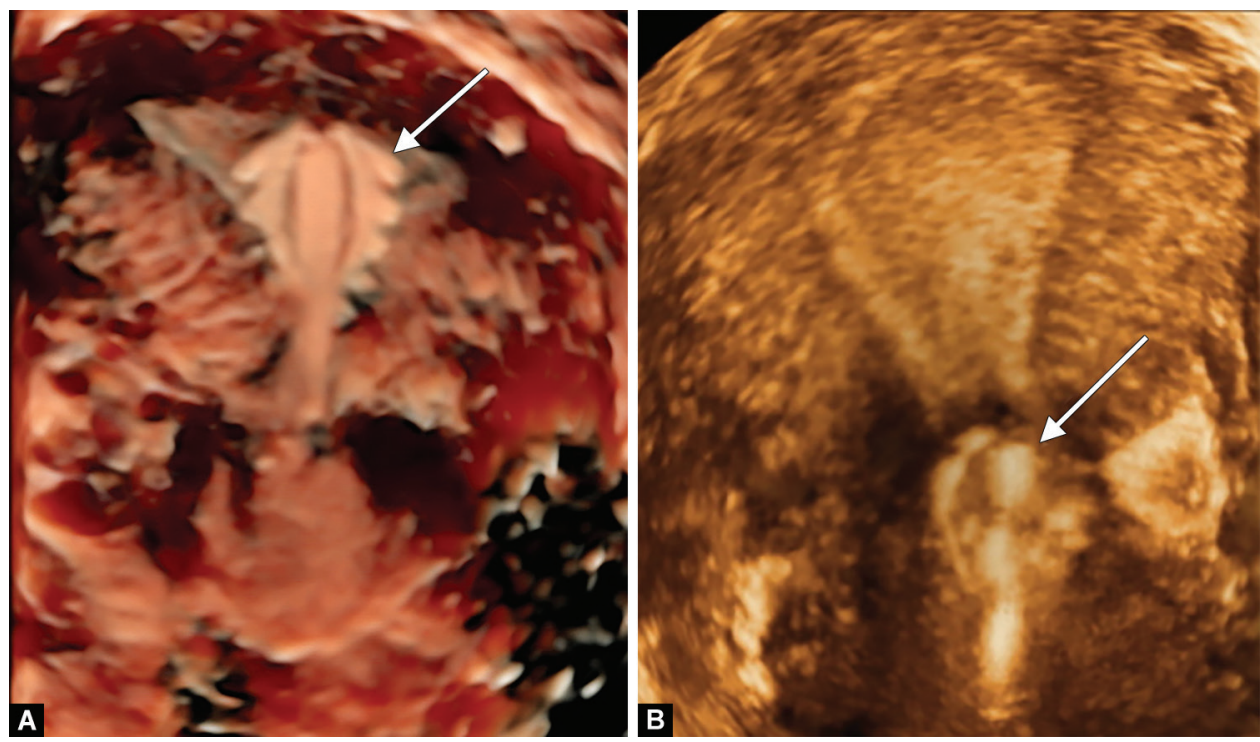

Figs $11 \mathrm{~A}$ and B: (A) 3D rendered ultrasound image showing normally positioned intrauterine device; (B) 3D ultrasound image showing displaced intrauterine device in the cervix

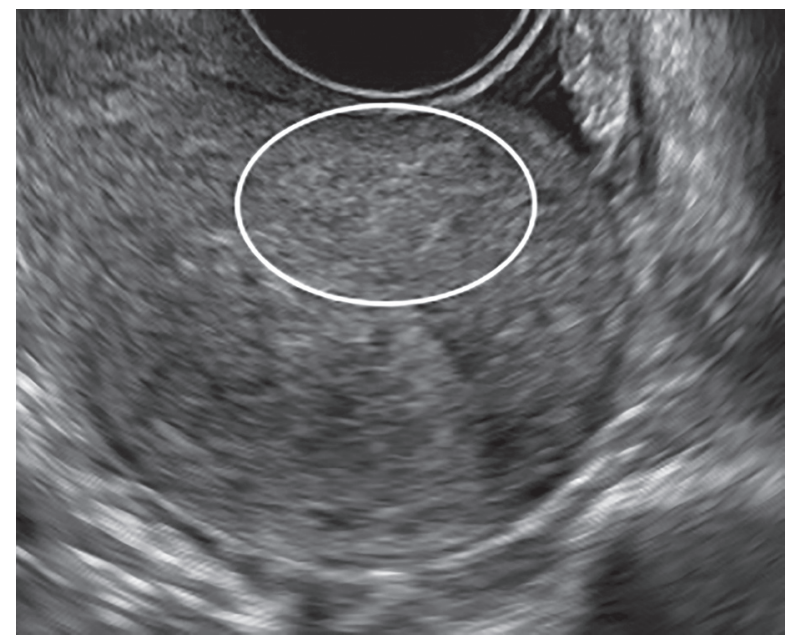

Fig. 12: B mode ultrasound image of uterus with adenomyosis (salt and pepper appearance)

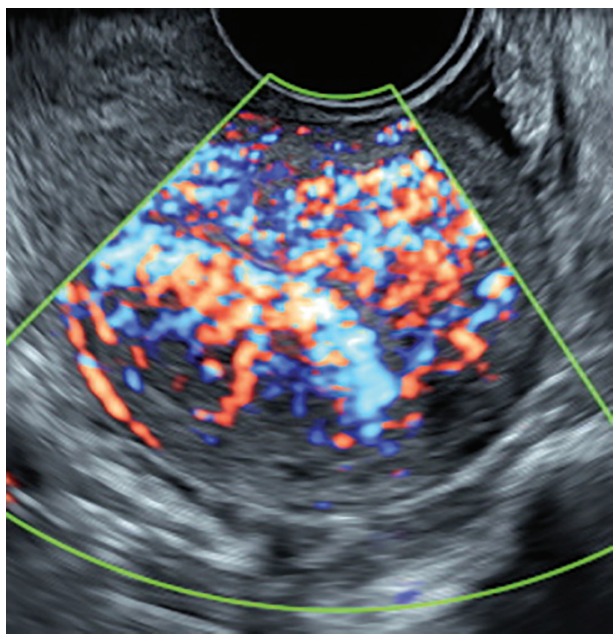

Fig. 14: HD flow in an adenomyotic uterus, showing dilated and translesional vascularity

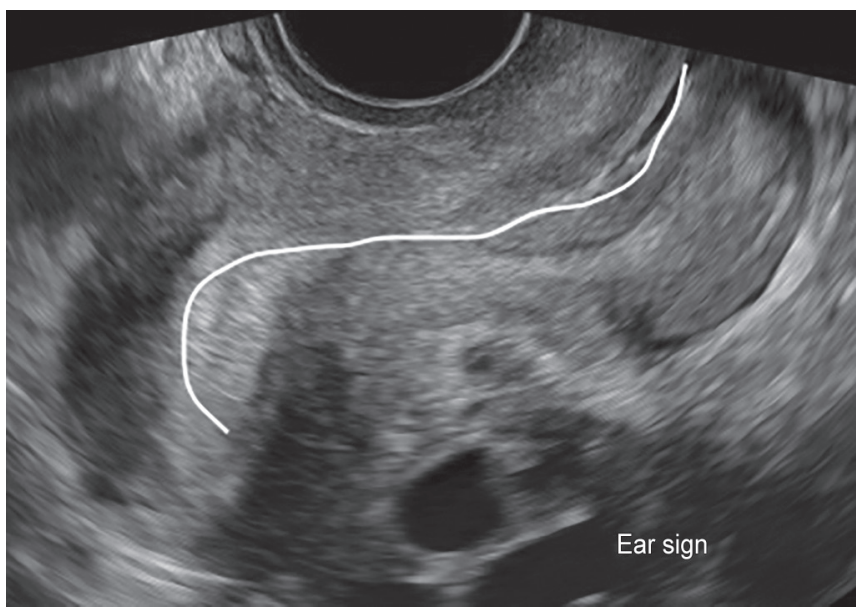

Fig. 13: B mode ultrasound image of adenomyotic uterus, demonstrating question mark sign of endometrium

\section{Adenomyosis}

This is a dichotomous disease characterized primarily by disruption of inner myometrial architecture and function, with secondary infiltration of endometrial elements into the myometrium. Patients present with dysmenorrhea, menorrhagia, dyspareunia, infertility, and recurrent abortions. Adenomyosis leads to derangement of normal myometrial peristalsis and blood supply and leads to immunological reaction leading to these symptoms. Prevalence of adenomyosis in infertile population is as high as $50 \%{ }^{2}$

On US, this typically shows thickening of the myometrium. This may be of one or both walls of the myometrium. The myometrium shows heterogeneous echogenicity with hyperechoic dots and lines. It also shows small anechoic myometrial cysts. The hyperechoic and anechoic areas together lead to a "salt and pepper" appearance (Fig. 12). The endometrial cavity typically may show an abnormal curvature in the fundal area and this is described as 'ear sign' or question mark sign (Fig. 13). The Doppler typically shows translesional vessels. The vessel diameter is more than that of normal spiral vessels (Fig. 14). The endometriomyometrial 
junction becomes irregular thick at places and is obliterated in other areas due to extension of the endometrial strands into the myometrium. This irregularity of the junctional zone is not well appreciated on B-mode US. Therefore, MRI was considered a superior modality for the diagnosis of adenomyosis. However, 3D US allows clear visualization of the junctional zone and also allows assessment of is thickness at different places in the uterus (Fig. 15). It is for this reason that all the parameters that were used for diagnosis of adenomyosis on magnetic resonance imaging (MRI), can also be used for 3D US also. Thickening of the transition zone thickness of $12 \mathrm{~mm}$ or greater has been shown to be associated with adenomyosis. The difference in the junctional zone thickness of $>4 \mathrm{~mm}\left(\mathrm{JZ}_{\text {diff }}>4 \mathrm{~mm}\right)$, JZ infiltration and distortion are also diagnostic criteria for adenomyosis. These have $88 \%$ sensitivity and, respectively, $85 \%$ and $82 \%$ accuracy $^{3}$ (Fig. 16). The junctional zone is can be measured on 3D US. The severity of irregularity is measured as the thickness of the thickest part of the junctional zone and thickness of the entire myometrial thickness. The magnitude of a $\mathrm{JZ}$ irregularity is expressed as the difference between the maximum and minimum JZ thickness: $\left(\mathrm{JZ}_{\text {dif }}\right)=\mathrm{J} \mathrm{Z}_{\max }-\mathrm{J} \mathrm{Z}_{\min } \cdot{ }^{4}$

Polypoid adenomyosis is seen as polyps with heterogenous echogenicity, well appreciated on 3D US. Its feeding vessel shows

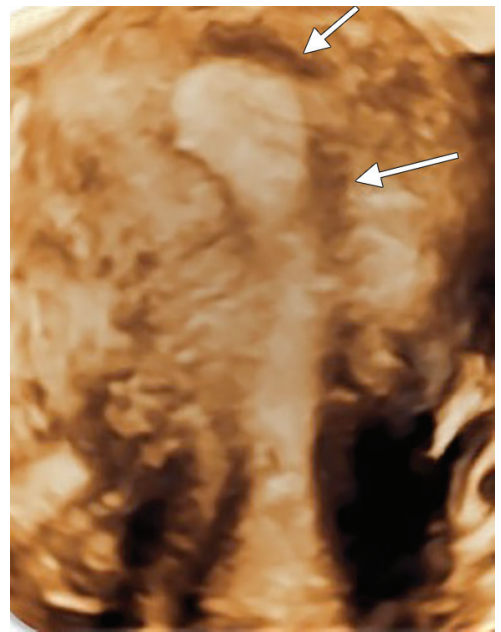

Fig. 15: 3D ultrasound rendered image of adenomyotic uterus showing irregular junctional zone
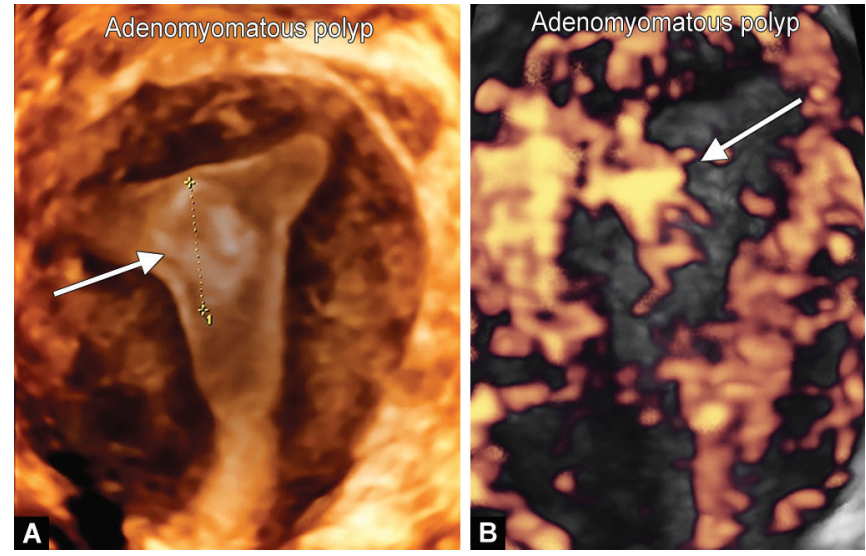

Figs 17A and B: (A) 3D ultrasound rendered image of the uterus showing adenomyomatous polyp; (B) 3D power Doppler angiorendered image of adenomyomatous polyp showing feeding vessel with abundant vascularity abundant branching inside the polyp and is most impressively demonstrated on 3D power Doppler (Fig. 17).

\section{Fibroids}

Fibroids are benign tumors made of fibrous and muscular tissue. As these are benign overgrowths, these displace the surrounding myometrial fibers and create a pseudocapsule. Therefore, fibroids are usually seen as well defined, roundish, and hypoechoic lesions. These are diagnosed on 2D US and may be subserosal, intramural, or submucosal. It is the later type in which 3D US has a role. Submucous fibroids are divided as T0, T1, and T2 depending on whether entire fibroid is inside the endometrial cavity, more than $50 \%$ is in the endometrial cavity or $<50 \%$ is inside the endometrial cavity. This distortion or invasion depends on the location and size of the fibroids. Moreover, a large fibroid in submucous location, which is distorting the endometrial cavity causes stretching of the endometrium and in turn compression of the blood vessels inside also. This hampers the endometrial blood flow required for the development of estrogen receptors and implantation and may cause subfertility. Three-dimensional US shows endometrial distortion by the fibroid very clearly (Fig. 18). This not only helps in

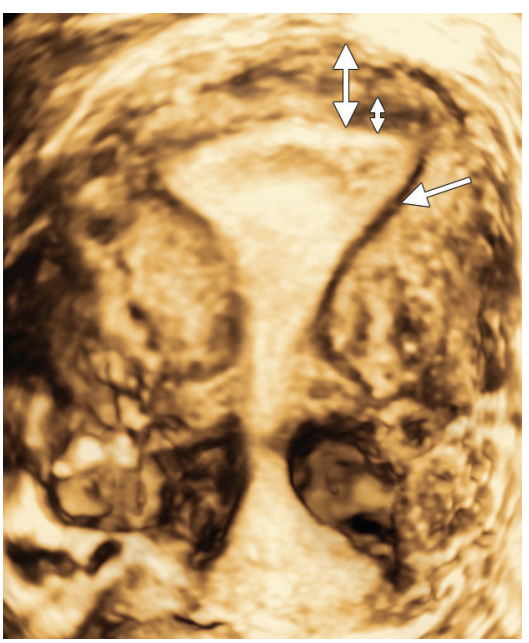

Fig. 16:3D ultrasound rendered image of the uterus showing irregularity of the junctional zone. The double headed arrows demonstrate the myometrial thickness and thickness of the irregular junctional zone. Single headed arrow shows the thickness of the normal junctional zone

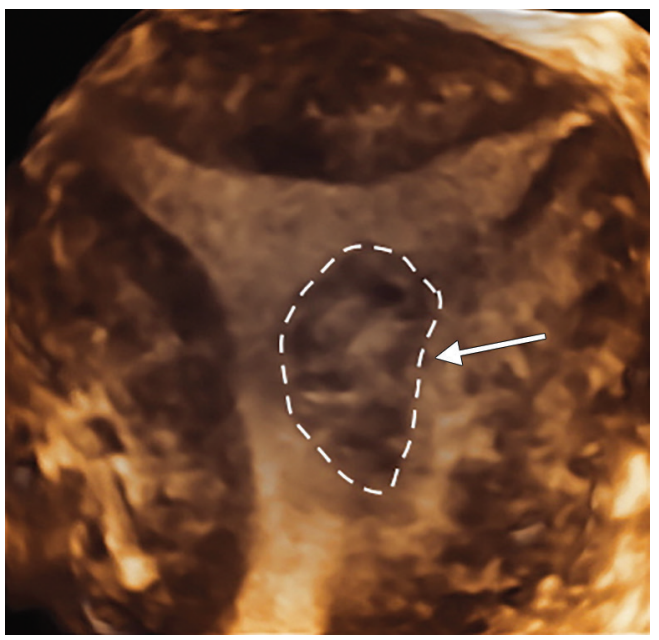

Fig. 18: 3D ultrasound rendered image of submucous fibroid as marked by dotted line showing distortion of the endometrial cavity 
deciding that the fibroid can be a cause of fertility problem or not but also helps to decide the route of surgery. A fibroid, the major part of which is popping in the endometrial cavity can be removed hysteroscopically and that of which only small part is projecting in the endometrial cavity has to be removed through the external surface of the uterus, either laparoscopically or by laparotomy.

Lasmar's score for submucous fibroids and STEPW classification that evaluates the size, topography, extension, penetration, and lateral wall involvement (submucous fibroids: a new presurgical classification (STEP-w) - Ricardo Lasmar, Paulo Barrozo, Rogeiro Dias e Marco A Oliveira) is being used widely for the same (Fig. 19). The scoring system is demonstrated in the pictures below.

Submucous fibroids are a frequent cause of menstrual abnormalities. Three-dimensional saline contrast hysteroscopy provides important information about the size and location of those fibroids. Submucous fibroid protrusion ratio, fibroid diameter, and size of fibroid's intramural component are significantly associated with the likelihood of successful hysteroscopic fibroid resection. ${ }^{5}$

Using 2D US and color Doppler usually, fibroids can be differentiated from adenomyomas as the former shows peripheral vascularity whereas the later shows translesional vascularity (Fig. 20). The edge shadows are seen in fibroids apart from the fan-shaped shadows, that are not seen in adenomyomas (Fig. 21). Differentiation is difficult when adenomyomas are relatively more defined or the fibroids show internal vascularity because of degeneration. Threedimensional power Doppler can be applied here to define the vascular arrangement in the lesion. A radial or irregular arrangement is suggestive of adenomyoma whereas a circular arrangement is suggestive of a fibroid (Fig. 22).

Fibroids may undergo malignant change when these are named as leiomyosarcoma. The pseudocapsule then shows discontinuity or is completely absent. It becomes difficult to define the extent of the lesion. It may invade the endometrium or may also extend not only up to serosa. Three-dimensional is a reliable tool to define the extent of the lesion, breaking of tissue planes, and involvement of other structures, using either rendering mode or tomographic US imaging in the scanning plane and in the coronal plane (Fig. 23). Moreover, extension of the malignant vascular pattern in the surrounding tissues may also be a reliable guide for the extension of the tumor.

A malignant lesion has neoangiogenesis and the vascular pattern is evidently different from the physiological angiogenesis. These are fast developing vessels, and therefore do not have muscularis layer in their walls and therefore show a low-resistance flow. These vessels distend/break easily under pressure thus forming microaneurysms and arteriovenous malformations. To cope up with the fast growing tumor, it also gives dichotomous branches. This creates a typical malignant chaotic pattern on 3D power Doppler which can be a very useful tool to differentiate benign from malignant lesions.

3D power Doppler signs of malignancy ${ }^{6}$ (Fig. 24).

- Loss of tree-like branching of vessels.

- Sacculation of arteries and veins.

- Focal narrowing of arteries.

- Internal shift of velocities within the arterial lumen,

- Beach-ball finding of abnormally increased and disorganized peripheral flow (a combination of above four characteristics). Beach ball appearance with a crowded haphazard interwoven matrix of arteries and veins suggest malignancy.

- Increased flow to a center of the solid lesion.

- Crowding of vascularity.
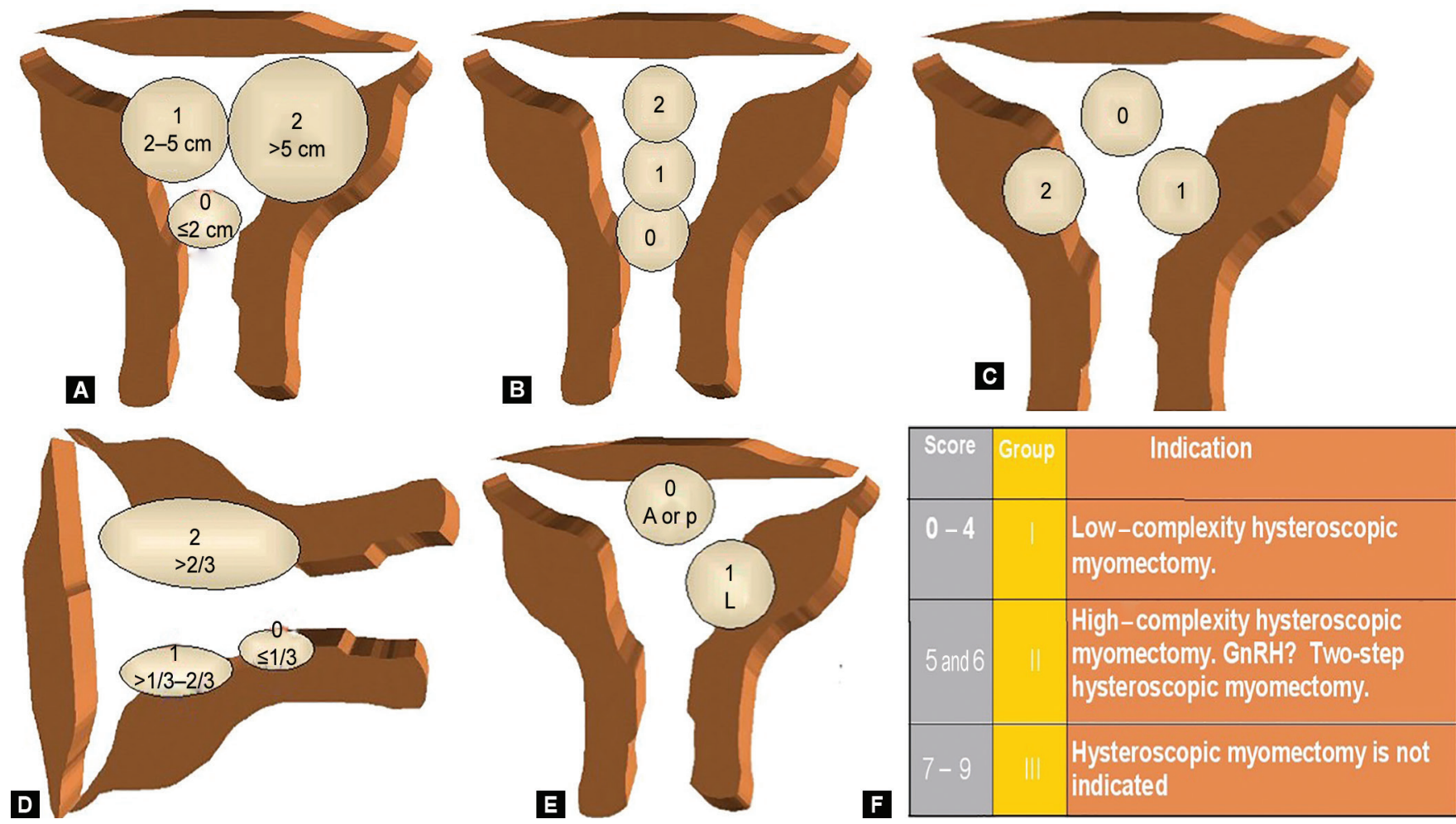

Figs 19A to F: (A to E) STEPW classification features for scoring size, topography, extension, penetration and lateral wall involvement; (F) Treatment protocol suggestions against a particular score 

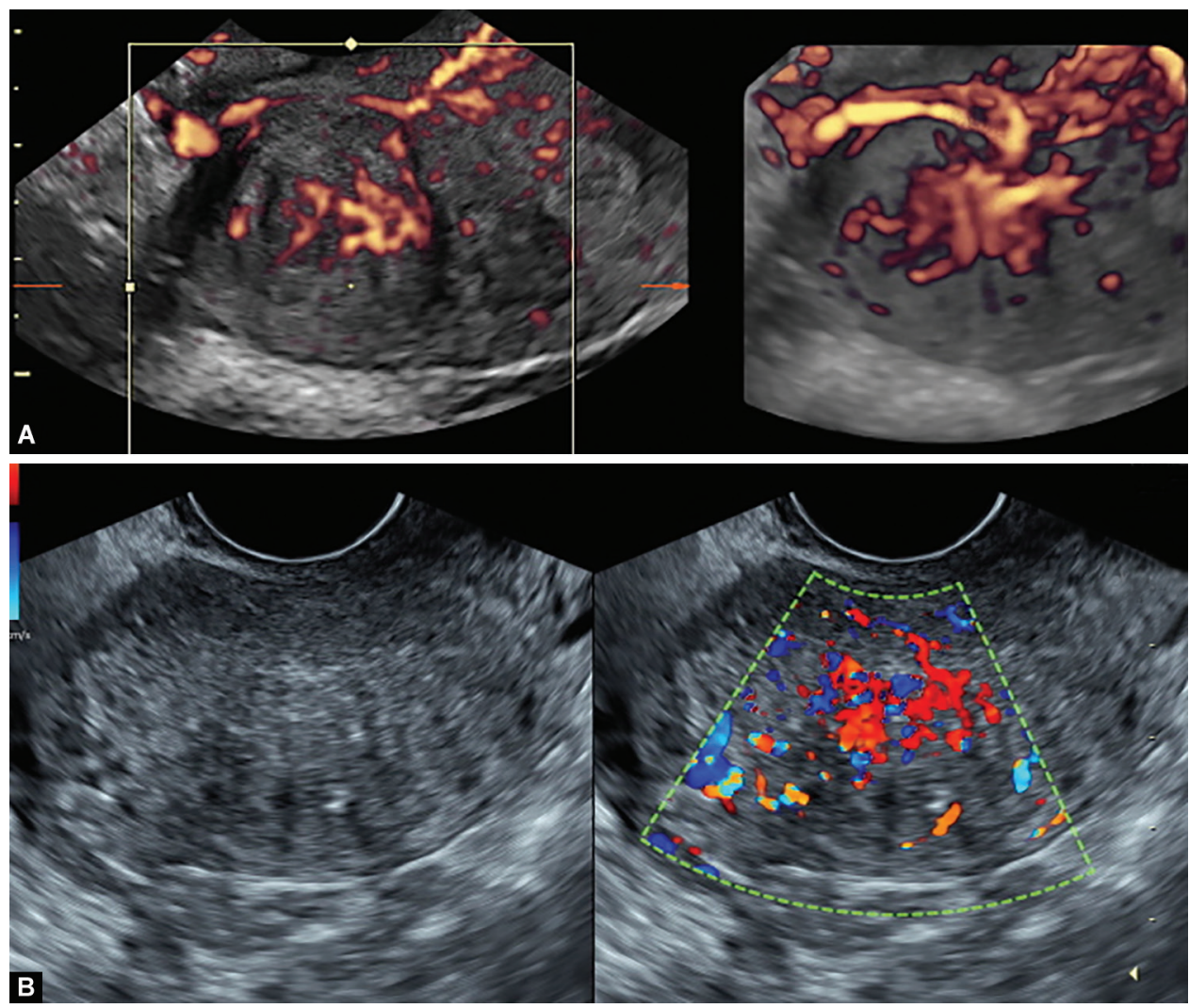

Figs 20A and B: (A) Power Doppler and 3D power Doppler image of a degenerated fibroid showing peripheral and intralesional vascularity; (B) B mode and color Doppler image of adenomyoma showing translesional vascularity

- Start and stop arteries: arteries appear to start and stop within a mass in a disjointed fashion, losing the tree-like the expected appearance of a benign mass.

Apart from lieomyosarcomas, this vascular pattern may also be a useful guide for diagnosis and extension of endometrial carcinoma also (Fig. 25).

\section{Adnexal lesions}

Most adnexal lesions are diagnosed by $B$ mode and Doppler and $3 D$ is not required. Though $3 D$ is used for demonstration of typical vascular pattern of the endometrioma (Fig. 26A). Endometriomas are known to have short coursed vessels in the periphery of the lesion. And this vessel tree when seen on 3D US, it typically shows a bird's nest appearance ${ }^{7}$ (Fig. 26B).

This is, of course, a supportive sign and not a diagnostic sign for endometrioma. Endometriomas on B mode usually show ground glass echogenicity, thick shaggy walls and hyperechoic flecks in the walls due to hemosiderin or cholesterol deposit ${ }^{8}$ (Fig. 27A). But endometriomas may appear as lesions with internal echogenicities other than like ground glass. The layering effect is commonly seen in endometriomas (Fig. 27B), due to blood in different forms due to new fresh bleeds and degeneration. Acoustic streaming is also often seen in endometriomas due to thick fluid content. ${ }^{9}$ Tenderness on probe pressure and adhesions are considered additional important supportive signs for the diagnosis of endometriomas.

As for the uterine malignancies, 3D power Doppler qualitative analysis of tumor angiogenesis allows a precise detection of the earliest appearance of ovarian malignancy-stage IA.

Differentiation between benign and malignant lesions by contrast-induced 3D PD. ${ }^{10,11}$

\begin{tabular}{ll}
\hline Sensitivity & $100 \%$ \\
Specificity & $93.9 \%$ \\
PPV & $85.7 \%$ \\
NPV & $100 \%$ \\
\hline
\end{tabular}

Tubal pathologies also are adnexal lesions. The one that is most commonly found in the infertile population is hydrosalpinx. Hydrosalpinx is distention of the fallopian tube due to inflammatory fluid and secretions as a result of obstruction at the fimbrial end most commonly but sometimes also involving the cornual end. This obstruction is due to adhesions and the commonest causes are the infection, endometriosis, and surgery. Hydrosalpinx may present as an extra-ovarian cystic lesion and it typically changes its shape on the rotation of the probe. It appears round in transverse section but sausage or retort shaped in the longitudinal section (Fig. 28). It shows thicker walls in acute inflammation (Fig. 29), often with ascites. Chronic inflammations show thinner walls and adhesions may also be seen. At times in chronic inflammation, tubes may appear rigid (Fig. 30). When the tube is markedly distended and tortuous, it may appear like multiple cystic areas. It is in these cases that 3D US aids to establish the continuity between these cystic areas and confirm the diagnosis of hydrosalpinx. Though coronal plane imaging either on multiplanar view or omniview (Fig. 31A) may be of help but in these cases rendering on minimum mode or inversion mode are the best alternatives (Fig. 31B) to establish the continuity between the cystic lesions and confirm the diagnosis of hydrosalpinx. 

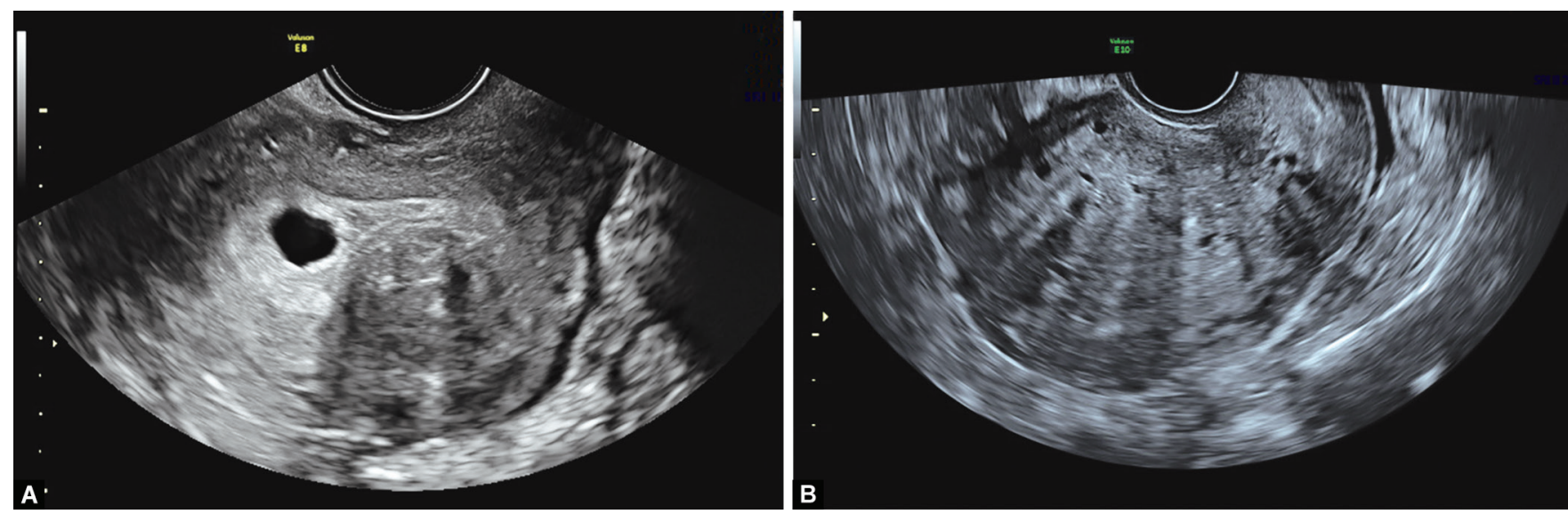

Figs 21A and B: (A) B mode ultrasound image showing fibroid with fan shadows and edge shadows; (B) Adenomyoma showing fan shadows
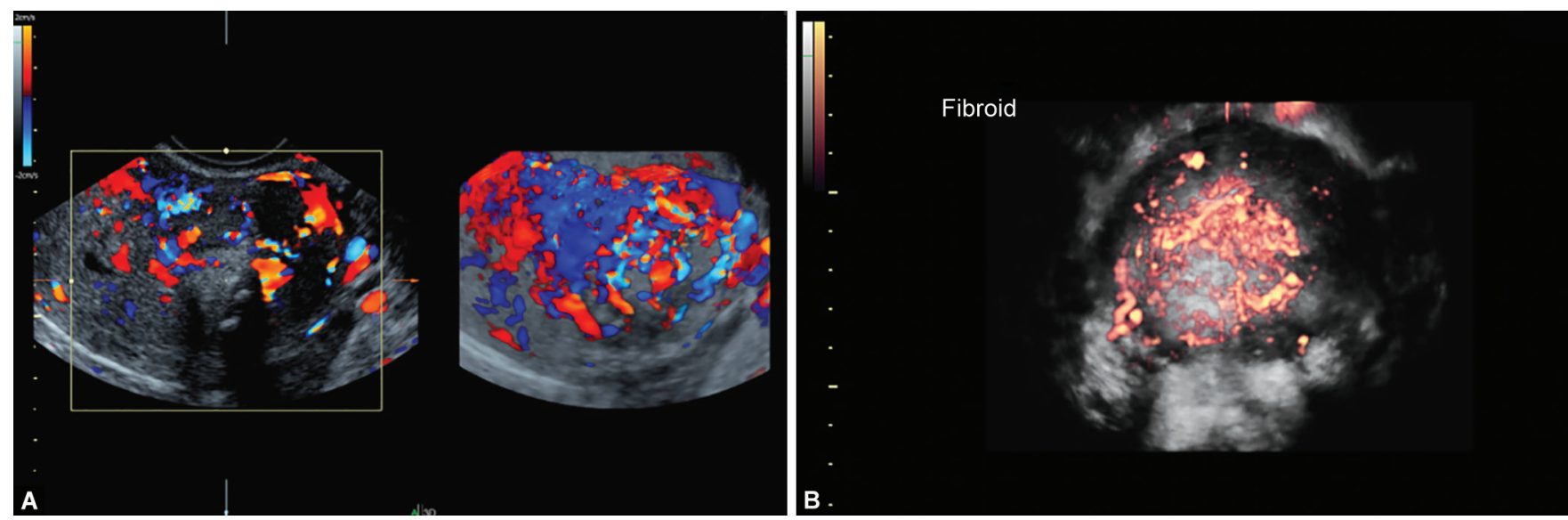

Figs 22A and B: (A) Color Doppler and 3D color Doppler image showing circular arrangement of blood vessels, suggestive of a fibroid; (B) 3D power Doppler glass body angioimage of adenomyoma showing irregular arrangement of the vessels

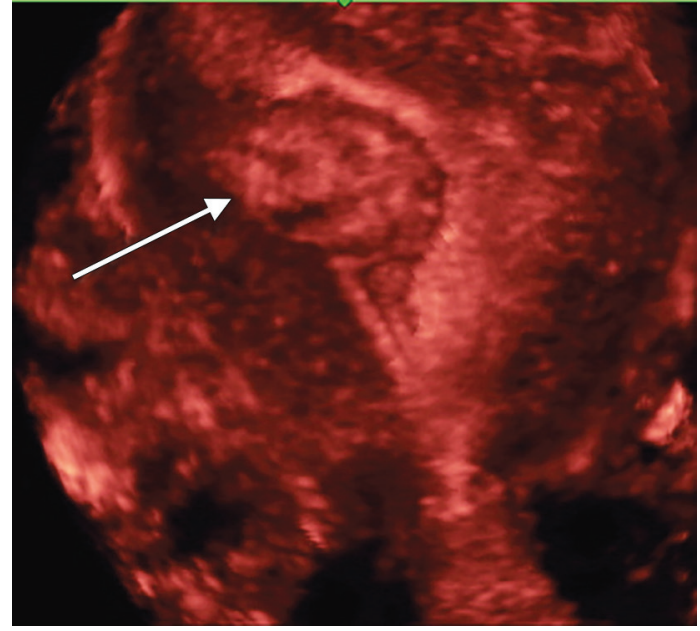

Fig. 23: 3D ultrasound rendered image of the uterus showing submucous fibroid with ill-defined lateral margin

\section{Tubal Patency Assessment}

In $25-35 \%$ of subfertile couples tubal pathology is a cause of subfertility. Consequently, assessment of fallopian tubes is an essential part of the subfertile female evaluation. There are various non-surgical and surgical techniques to assess fallopian tubes.

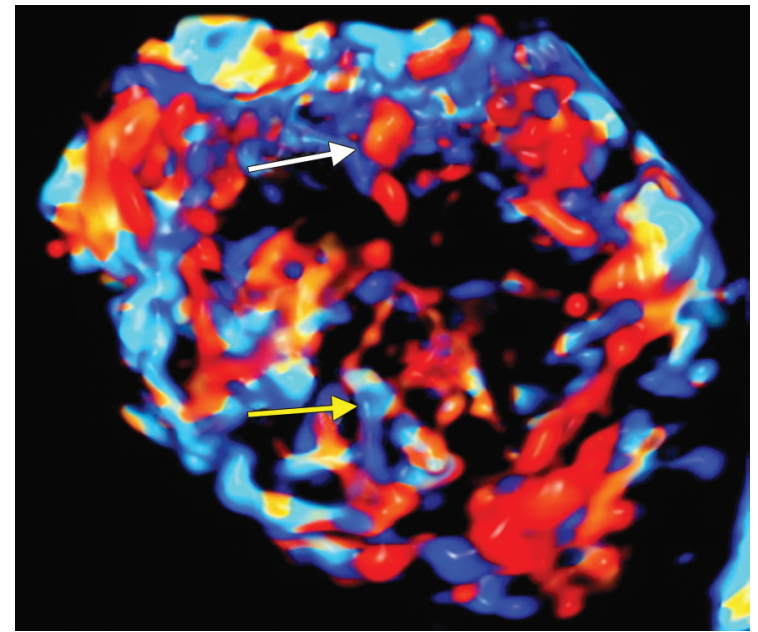

Fig. 24: 3D angiography showing vessels with irregular caliber (white arrow), and vascular communications (yellow arrow) suggestive of malignant features of the vascular tree

Nonsurgical techniques like hysterosalpingogram (HSG) (Carey, 1914) and US-based techniques such as saline infusion sonography (Corfman \& Taylor, 1966) and hystero-contrast-sonosalpingography (HyCoSy) (Deichert, 1993) are less invasive and there is less risk than in surgical technique, such as laparoscopy and dye test 
(chromopertubation). To demonstrate lumen of tubes, it is necessary to visualize the movement of fluid using highly echogenic medium. ${ }^{12}$

Hyperechogenic contrast medium enhances echo signals and enables detection of the flow by both B mode and Doppler US. Hysterocontrast-sonosalpingography is a very safe and relatively low-cost outpatient procedure. Its accuracy has been assessed in meta-analysis where we compared results of HyCoSy and laparoscopy and dye tests in 428 infertile women. As a result, we found the sensitivity of $93.3 \%$ and specificity of $89.7 \% .^{13}$

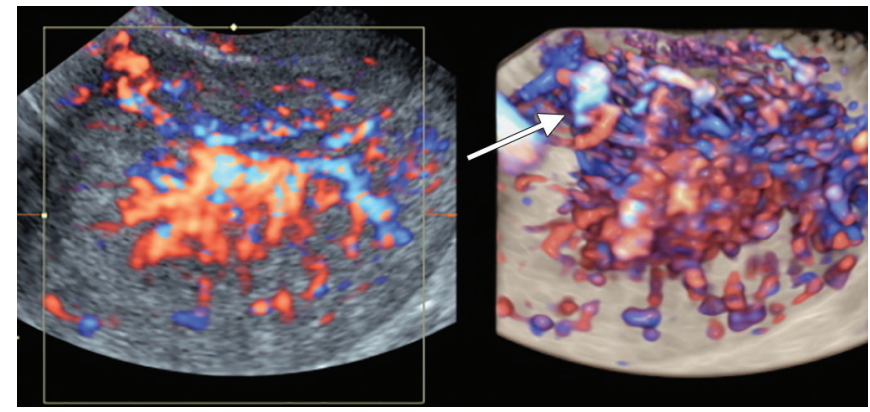

Fig. 25: HD flow and 3D HD flow image of the endometrial carcinoma extending into the anterior myometrium as diagnosed by extension of the malignant vascular pattern into myometrium as marked by arrow
In a study by Exacoustas et al., it was shown as well that HSG and HyCoSy had the same high concordance as laparoscopy- $-86.7 \%$ and $86.7 \%$, respectively. ${ }^{14}$ Three-dimensional power Doppler helps, therefore, to try to visualize the whole tube and spill. This technique was found to be superior to conventional HyCoSy with a free spill from fallopian tubes which was demonstrated in $91 \%$ of tubes using 3D power Doppler as compared to only $46 \%$ by conventional $\mathrm{HyCoSy}$ while the required contrast agent was almost half for 3D PD in one of this study ${ }^{15}$ (Fig. 32).

There are various advantages with 3D HyCoSy techniques. They allow simultaneous visualization of the uterine cavity and the whole tube, short procedure time, and reduced patient's discomfort. They require less amount of contrast and storage of the 3D volume, which allows later off-line review and reassessment (Fig. 33).

\section{Advantages of 3D HyCoSy}

- Uterine cavity and whole tube can be seen together.

- Condition of lumen and fimbriae can be studied.

- Shortens procedure time and patient discomfort.

- Less amount of contrast is required.

- Storage of volumes allows reassessment and reviews.

- Spill is better appreciated.

- More accurate.
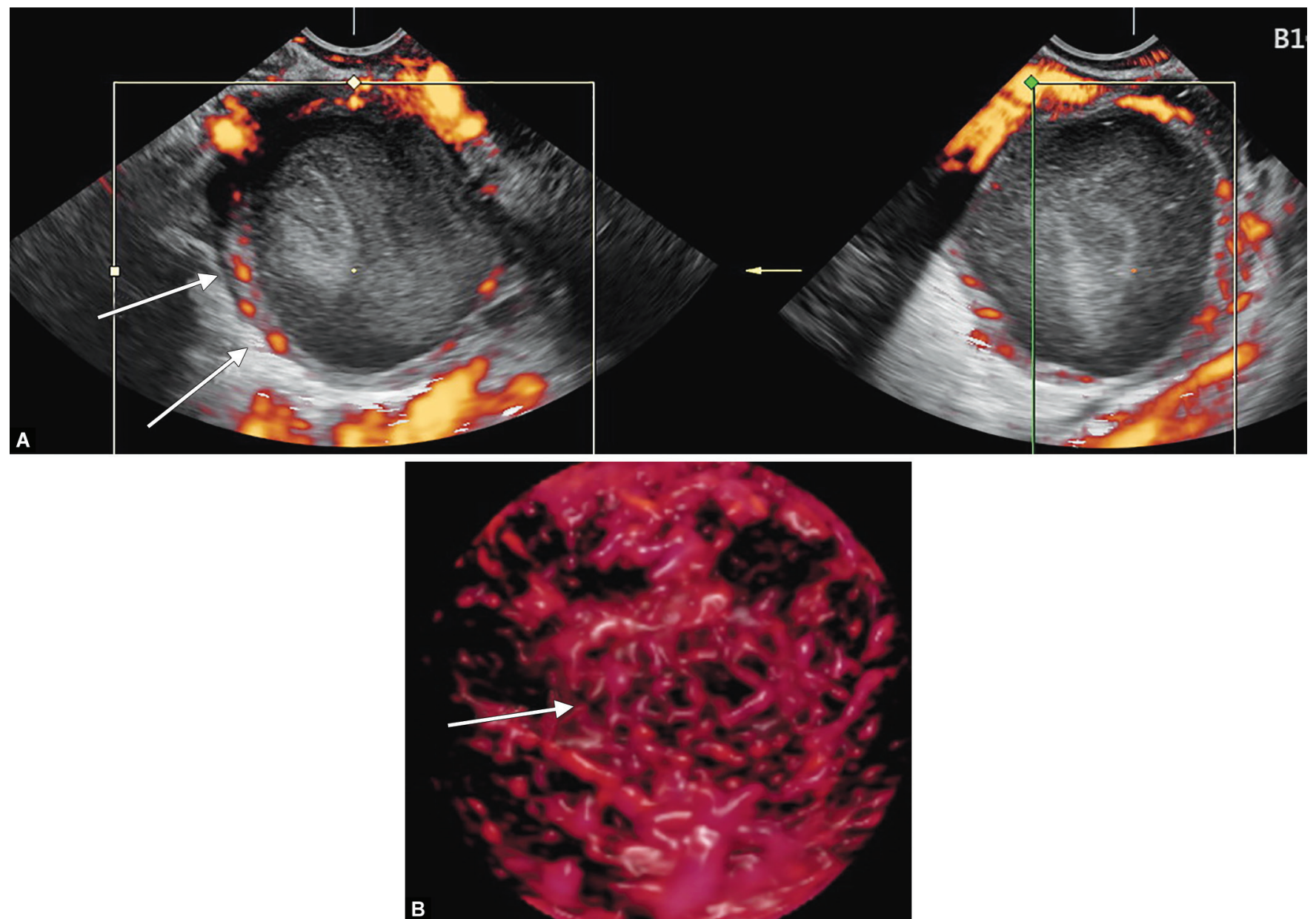

Figs 26A and B: (A) Power Doppler image of endometrioma showing short coursed vessels; (B) 3D power Doppler of the endometrioma showing bird's nest appearance 

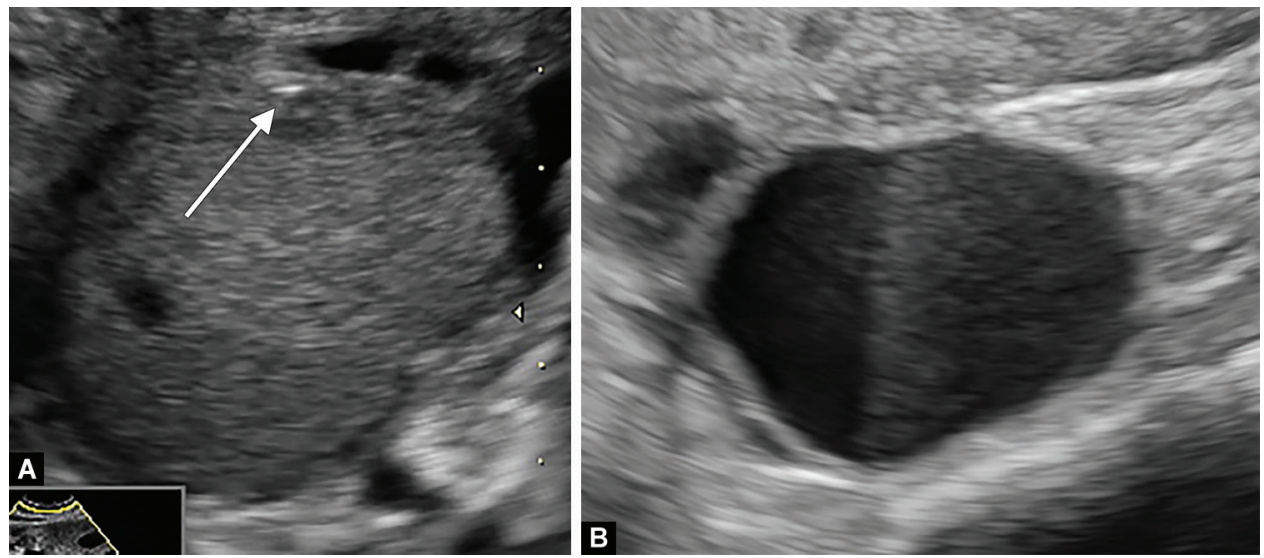

Figs 27A and B: (A) B mode ultrasound image of endometrioma with ground glass echogenicity and hyperechoic fleck (arrow) in the wall; (B) Vertical fluid-fluid level of the endometrioma as seen on B mode image

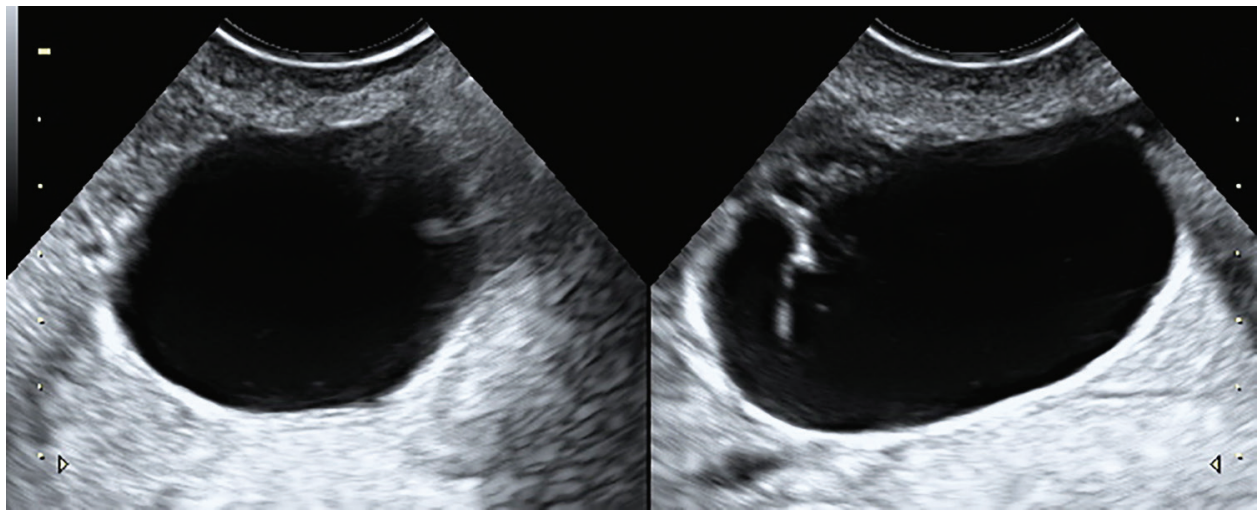

Fig. 28: B mode ultrasound image of hydrosalpinx in transverse and longitudinal sections

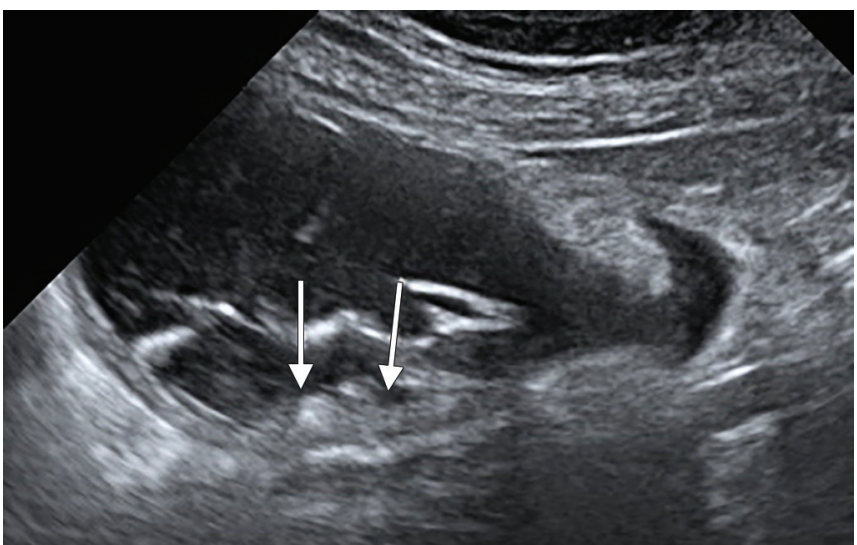

Fig. 29: B mode image of acute hydrosalpinx showing thick walls due to acute inflammatory edema

\section{Technique of 3D Hycosy}

Sonovue (Bracco) was used as the contrast agent. Preparation of the patient, catheter placement, and preparation of contrast media was done according to before mentioned method. Scanning is performed using a 3D US machine (e.g., Voluson E8 Expert BT 12; GE medical Systems). A high frequency (6-9 MHz) transvaginal volume probe is used for pelvic evaluation. Contrast mode is switched on the machine. While the contrast is slowly injected through the balloon catheter into the uterus, the transvaginal probe is so

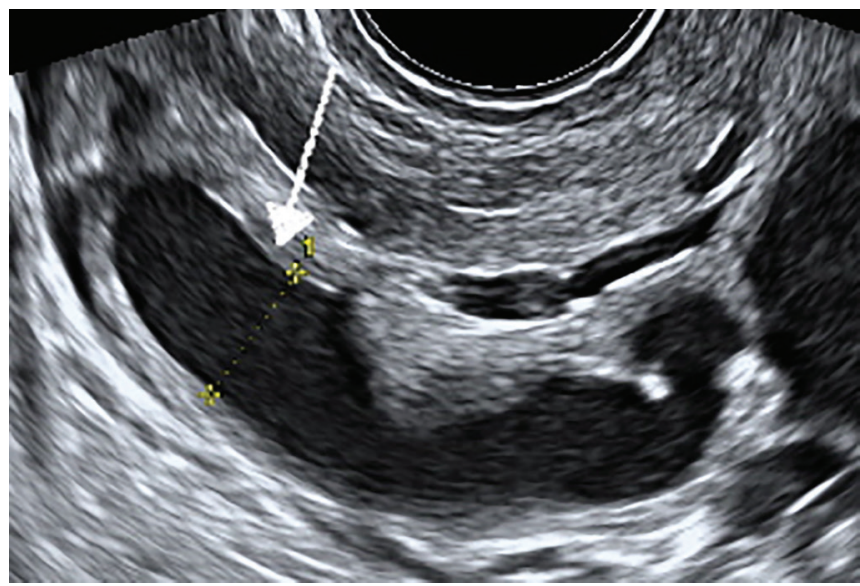

Fig. 30: Chronically inflamed tube with hydrosalpinx showing thin walls and only mild dilatation due to rigid walls

oriented that uterine cornu and ovaries are seen on the same plane. Having defined the contrast filling in the tubes, 3D is switched on and volumes are acquired for each side independently (Fig. 34).

Rendering is done in front back viewing direction, the surfaceenhanced mode is used, and the threshold is set to make the contrasting path more obvious. Magicut (electronic scalpel) is then used to cut all shadows other than the contrasting path. Then HD live rendering mode is switched on and the direction of 

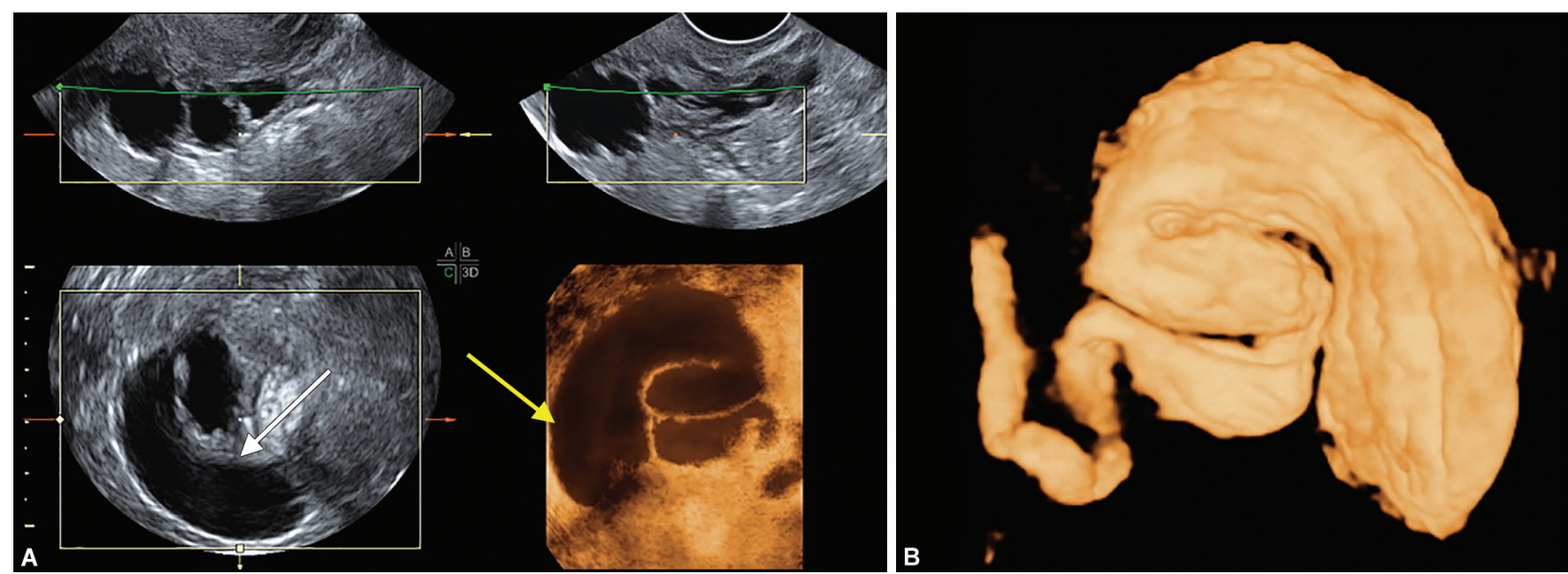

Figs 31A and B: (A) 3D ultrasound acquired multiplanar image of hydrosalpinx, seen as multiple cystic lesions on the acquired plane but on the coronal plane the continuity of these lesions is seen as marked by white arrow and on minimum mode imaging the entire tube is seen (yellow arrow); (B) The same lesion as mentioned in figure $A$ is shown in inversion mode

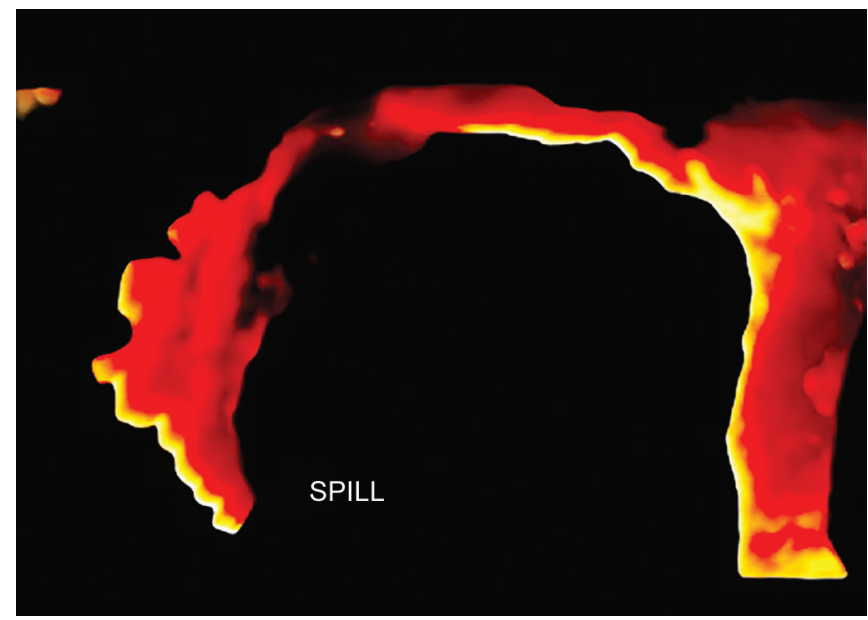

Fig. 32: 3D power Doppler hysterocontrast-sonosalpingography, showing uterus and right fallopian tube

the light is adjusted to visualize the fimbriae and spill to its best. After the final picture is ready, both the halves are matched and put together to make a complete picture of the uterus and both tubes (Fig. 35).

Hysterocontrast-sonosalpingography (HyCoSy) with automated 3D- technology retains the advantages of conventional 2D HyCoSy while overcoming the disadvantages. Two-dimensional HyCoSy is highly observer-dependent and it is only accurate in the hands of experienced investigators. When obtaining a volume of the uterus and tubes, automated 3D volume acquisition enables visualization of the tubes in the coronal view and of the tubal course in 3D space. This allows to less experienced operators to evaluate tubal patency status relatively easily. ${ }^{16}$

Large studies reported that 3D HyCoSy is highly accurate giving $100 \%$ sensitivity, $67 \%$ specificity, $89 \%$ positive predictive value (PPV), and $100 \%$ negative predictive value (NPV) for tubal patency and the concordance rate with laparoscopy of $91 \%{ }^{17}$

In a study by Kupesic et al., 3D HyCoSy (sensitivity, specificity, PPV, and NPV of $97.9 \%, 100 \%, 97.9 \%$, and $100 \%$, respectively) was learned to be marginally superior to 2D HyCoSy (sensitivity,

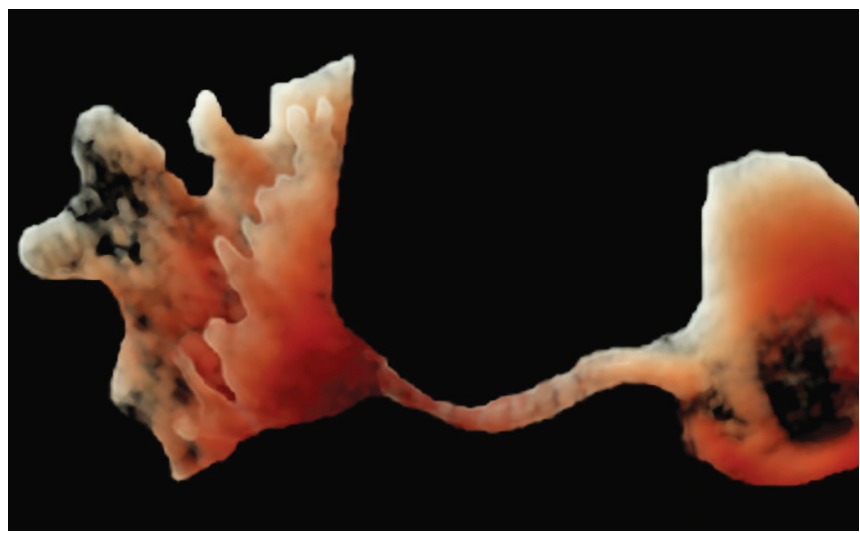

Fig. 33: 3D HYCoSy rendered in HD live mode shows the uterus, right fallopian tube with the fimbrial end

specificity, PPV, and NPV of 93.6\%, 97.3\%, 98.2\%, and 97.3\%, respectively) for tubal assessment. ${ }^{18}$

Three-dimensional SonoVue-HyCoSy showed a sensitivity of $93.5 \%$, specificity of $86.3 \%$, positive and negative predictive values of $87.8 \%$ and $92.6 \%$, respectively, and with a diagnostic accuracy of $90.0 \%$. The test-positive rates of 3D SonoVue-HyCoSy vs laparoscopy and dye were not significantly different $(82 / 150$ vs $77 / 150, P>0.05){ }^{19}$

Yet another study by Chan et al. has shown that the sensitivity of 3D-HyCoSy for detecting tubal patency was $100 \%$ with a specificity of $67 \%$. Positive and negative predictive values were $89 \%$ and $100 \%$, respectively. The concordance rate was $91 \%$. The mean duration $( \pm S D)$ for the 3D-HyCoSy was $13.4 \pm 5.5$ minutes. ${ }^{17}$ Color-coded 3D-PDI with surface rendering allowed visualization of the flow of contrast through the entire tubal length. The free spill of contrast was clearly identified in the majority of cases. The 3D-power Doppler imaging (PDI) method appeared to have advantages over the conventional HyCoSy technique, especially in terms of visualization of spill from the distal end of the tube, which was achieved twice as often with the 3D technique. The 3D-PDI technique allowed better storage of the information for re-analysis and archiving than conventional HyCoSy. The mean duration of the 


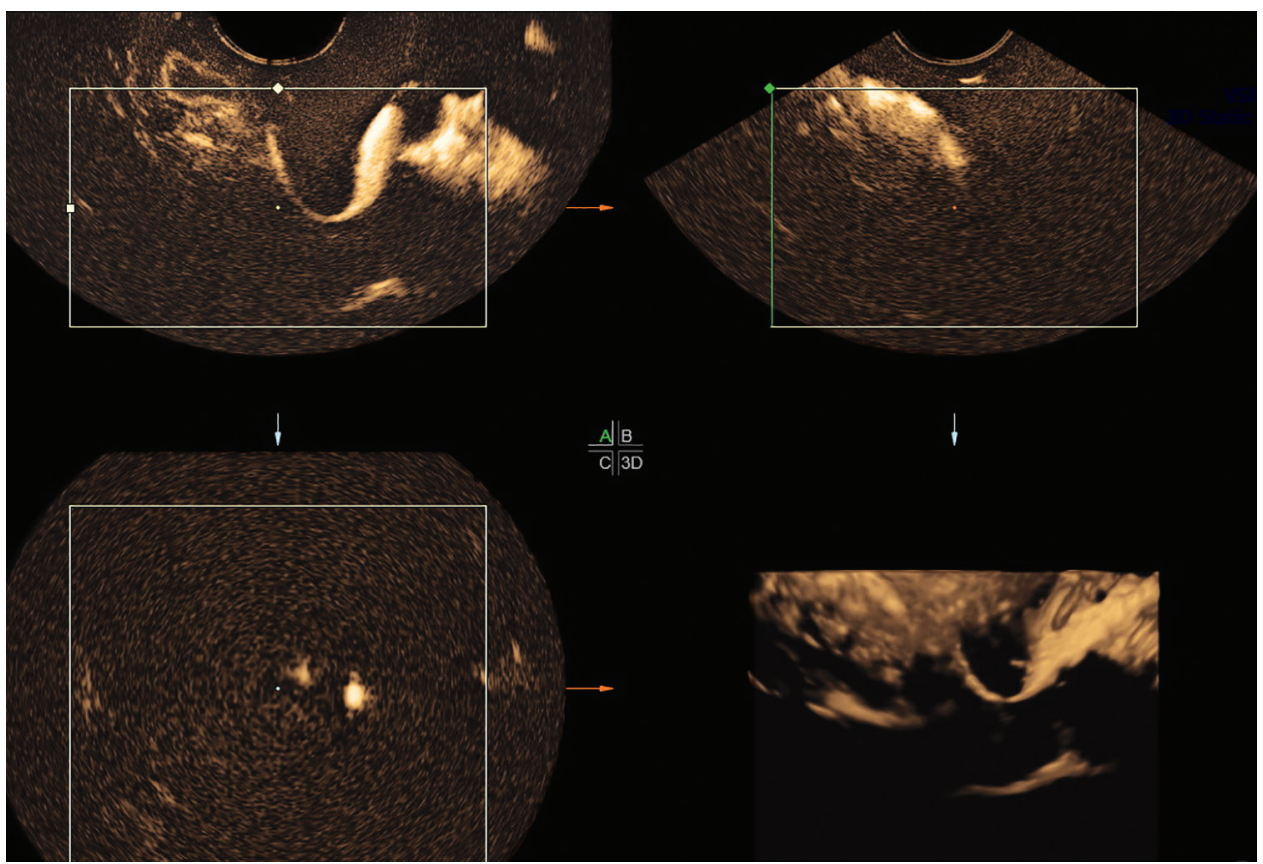

Fig. 34: 3D ultrasound acquired image of the uterus and tubes with hysterocontrast-sonosalpingography and contrast mode

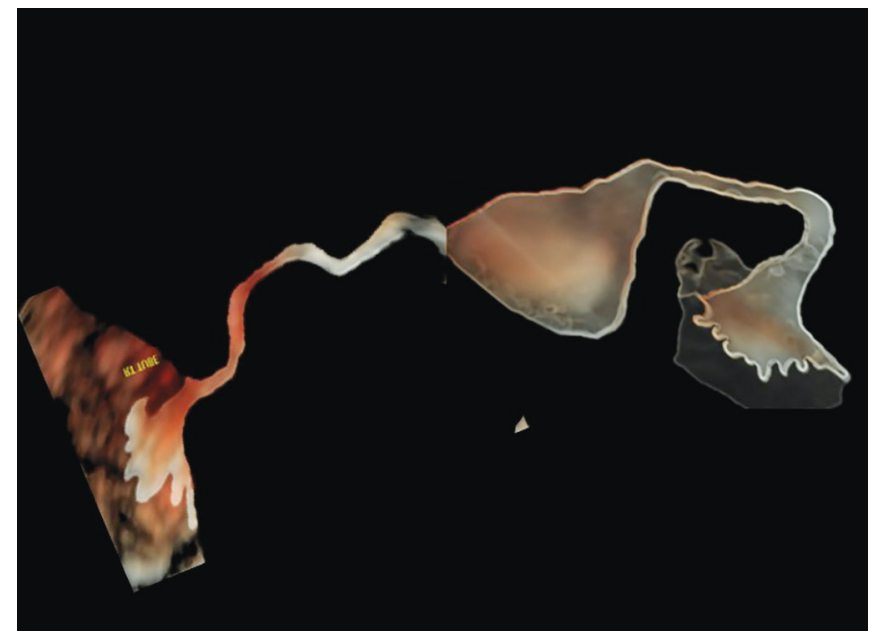

Fig. 35: HD live rendered image of the 3D HyCoSy, reconstructed for one tube and uterus at a time and pasted together on the computer

imaging procedure was less with 3D-PDI, but the operator time, which included post-procedure analysis of the stored information, was similar. A significantly lower volume of contrast medium $(5.9 \pm 0.6 \mathrm{~mL})$ was used for 3D-PDI in comparison with that $(11.2 \pm 1.9 \mathrm{~mL})$ used for conventional $2 \mathrm{D}$ HyCoSy. ${ }^{20}$

\section{Polycystic Ovaries}

According to the European Society of Human Reproduction and Reproductive Endocrinology (ESHRE)/American Society of Reproductive Medicine (ASRM) consensus 2018, the polycystic ovarian syndrome (PCOS) consists of various different phenotypes and in majority US showing polycystic ovaries was important.

Polycystic ovaries (PCO) on US according to this consensus is an ovary that is $10 \mathrm{cc}$ in volume and/or has more than 20 antral follicles of 2-9 mm in diameter. But this criteria of $>10 \mathrm{cc}$ would fail to identify a group of ovulatory, normo-androgenic women still at risk of complications associated with PCOS, such as ovarian hyperstimulation syndrome (OHSS), failed implantation, miscarriage, and hyperinsulinemia. ${ }^{21}$ The ovarian volume of $6.6 \mathrm{cc}$ has $91 \%$ sensitivity and $91 \%$ specificity for PCOS. ${ }^{22}$ Jonard et al. have also suggested that lowering the ovarian volume to $7 \mathrm{cc}$ has allowed $67.5 \%$ sensitivity and $91.2 \%$ specificity for PCOS. And the mean ovarian volume in normal ovaries was suggested as $4.5 \mathrm{cc}$ in this study. ${ }^{23}$ But it is important to assess the correct ovarian volume and this can be done by 3D US. After the volume of the ovary is acquired by 3D US, virtual organ computer-aided analysis (VOCAL) software is used to calculate the ovarian volume. Virtual organ computeraided analysis calculates the volume of any structure by rotating it $180^{\circ}$. A rotating step of $6^{\circ}-30^{\circ}$ can be selected. A circumference is drawn around the structure of interest at every step of rotation and at the end of $180^{\circ}$, the total volume is calculated by the scanner computer (Fig. 36).

But size is not the only important criteria. Histologically and biochemically polycystic ovaries can be of normal size and a wide overlap is seen between the size of normal and polycystic ovaries. Polycystic ovarian morphology has been found to be a better discriminator than ovarian volume between polycystic ovarian syndrome and control women. ${ }^{24}$ Amongst the morphological features of polycystic ovaries are the follicular number per ovary (FNPO) of more than 12 according to the old consensus and more than 20 per ovary according to a new consensus, the follicular arrangement that may be peripheral or generalized and stromal abundance.

The mean FNPO of follicles $2-5 \mathrm{~mm}$ in size was significantly higher in polycystic ovaries than in controls, while it was similar within $6-9 \mathrm{~mm}$ range. Within $2-5 \mathrm{~mm}$ range, a significant relationship was found between FNPO and androgens but FNPO in the range of 6-9 $\mathrm{mm}$ was significantly and negatively related to body mass index and fasting serum insulin level. It is believed that androgen leads to early follicular development but the further progression is not normal due to hyperinsulinemia and/or other metabolic influence linked to obesity. ${ }^{25}$ Androgen is the cause and 


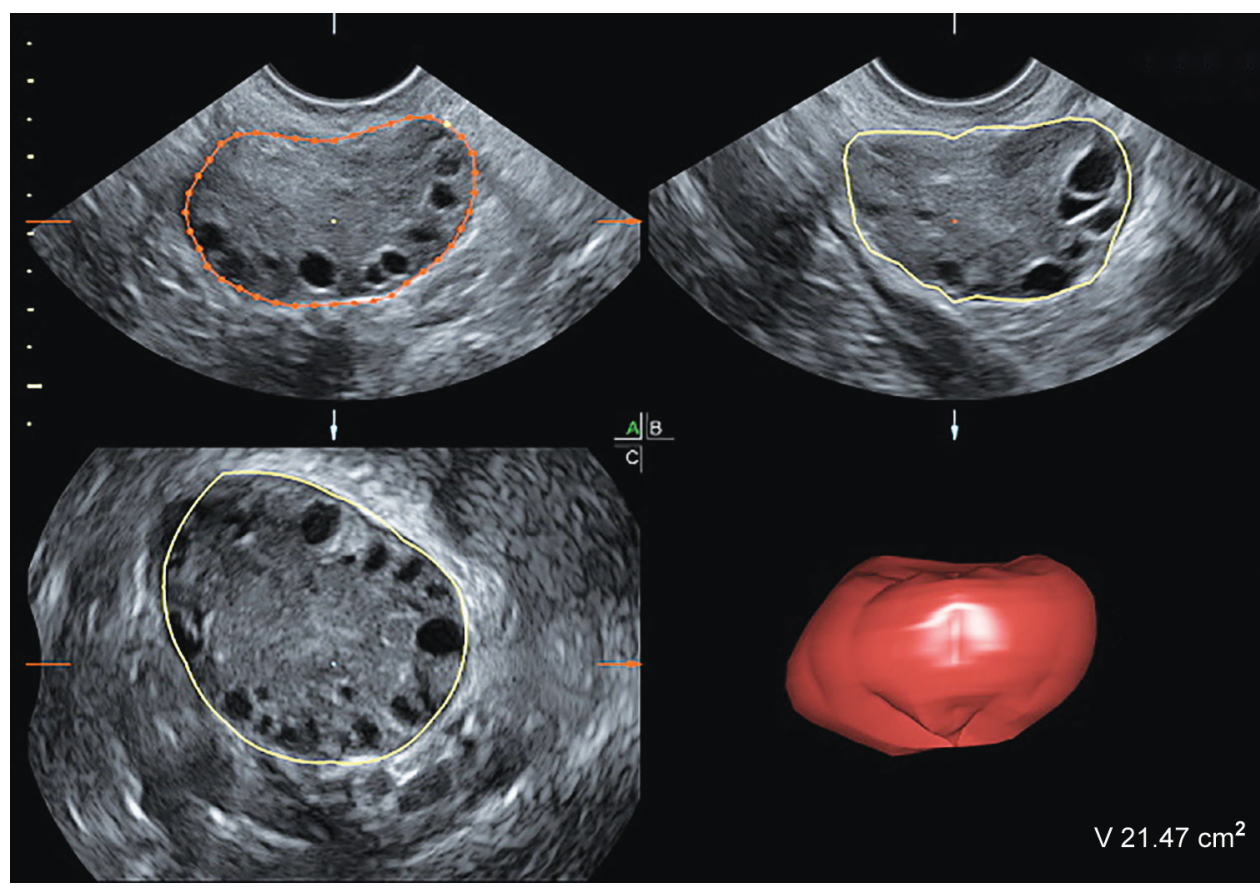

Fig. 36: Virtual organ computer-aided analysis calculated volume of the ovary on 3D ultrasound acquired volume of ovary
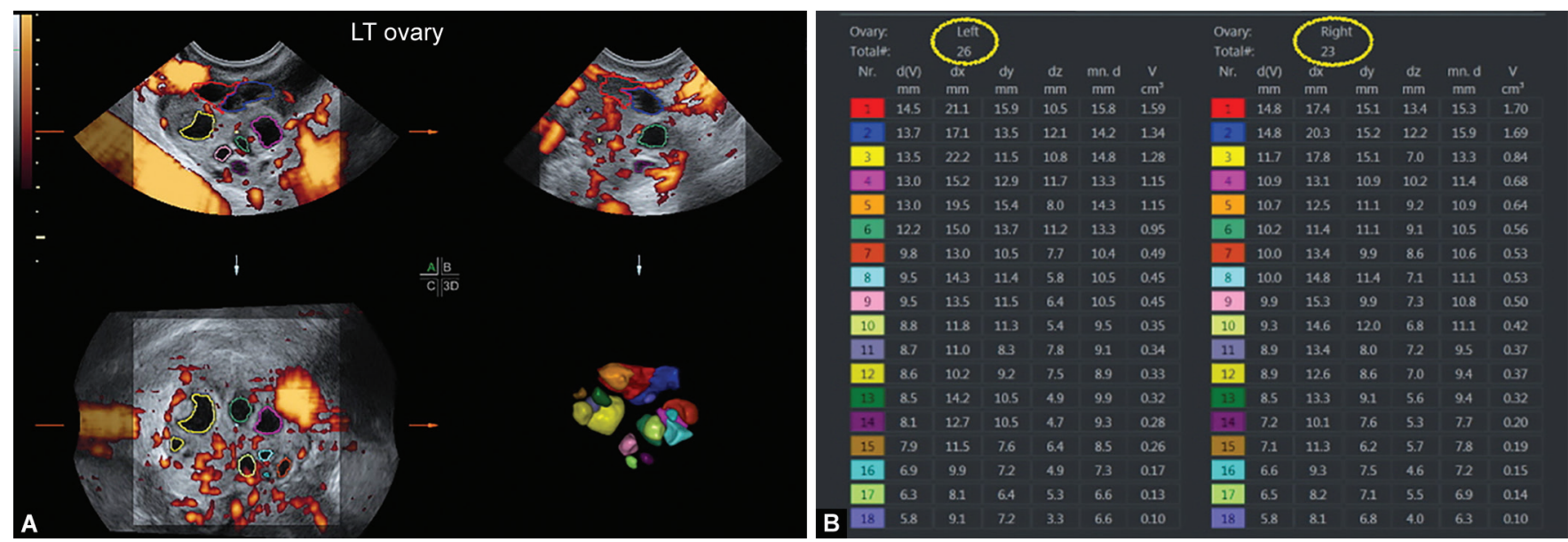

Figs 37A and B: (A) Three-dimensional ultrasound acquired volume of the ovary, with SonoAVC showing color-coded follicles; (B) Result sheet of SonoAVC showing total number of follicles in either ovary (circles) and all three diameters, mean diameter and volume of individual follicle marked by the relevant color in the box

$\mathrm{AMH}$ is the result of multiple antral follicles. An average value of 26 or more follicles per ovary is a reliable threshold for detecting polycystic ovaries in women with a frank manifestation of PCOS. Sensitivity and specificity for the diagnosis of PCOS for FNPO (26) was $85 \%$ and $94 \%$, respectively, and for ovarian volume (OV) (10 cc) was $81 \%$ and $84 \%$, respectively. ${ }^{26}$

These antral follicles that are more than 12-20 can be confidently counted only by 3D US and Sonographic automated volume calculation (SonoAVC) (Fig. 37A). SonoAVC is based on the inversion mode and color codes the follicles thus allowing more correct calculation of the number. The more exact value of antral follicle count (AFC) was acquired when counted by 3D US. A number of follicles $>12 \mathrm{~mm}$ on day of oocyte retrieval correlated significantly with AFC counted by 3D US rather than 2D US. Inversion mode is the most convenient method for counting antral follicles when they are multiple. ${ }^{27}$ SonoAVC measured less number of follicles on
AFC, though it took significantly less time to measure the size and record the number of antral follicles $(132 \pm 56.23$ seconds vs $324.47 \pm$ 162.22 seconds). ${ }^{28}$ Post-processing can be used for the follicles that are missed out on automated counting. Apart from this SonoAVC also calculates the size of each follicle and that is important for the understanding of the variable biochemical derangement in various patients with PCOS (Fig. 37B).

Stromal abundance the second important feature of polycystic ovaries. The earliest description of polycystic ovaries appears to date from 1845 as "sclerocystic ovaries". ${ }^{29}$ To identify women with a milder form of PCOS, further information, particularly about the ovarian stroma and the degree of vascularization, is required. ${ }^{21}$ Patients having long standing PCOS and long standing anovulation have more dense stroma and a cardinal feature has been shown to be the presence of a bright, highly echogenic stroma on transvaginal US. ${ }^{30}$ Stromal hypertrophy was recognized as a 


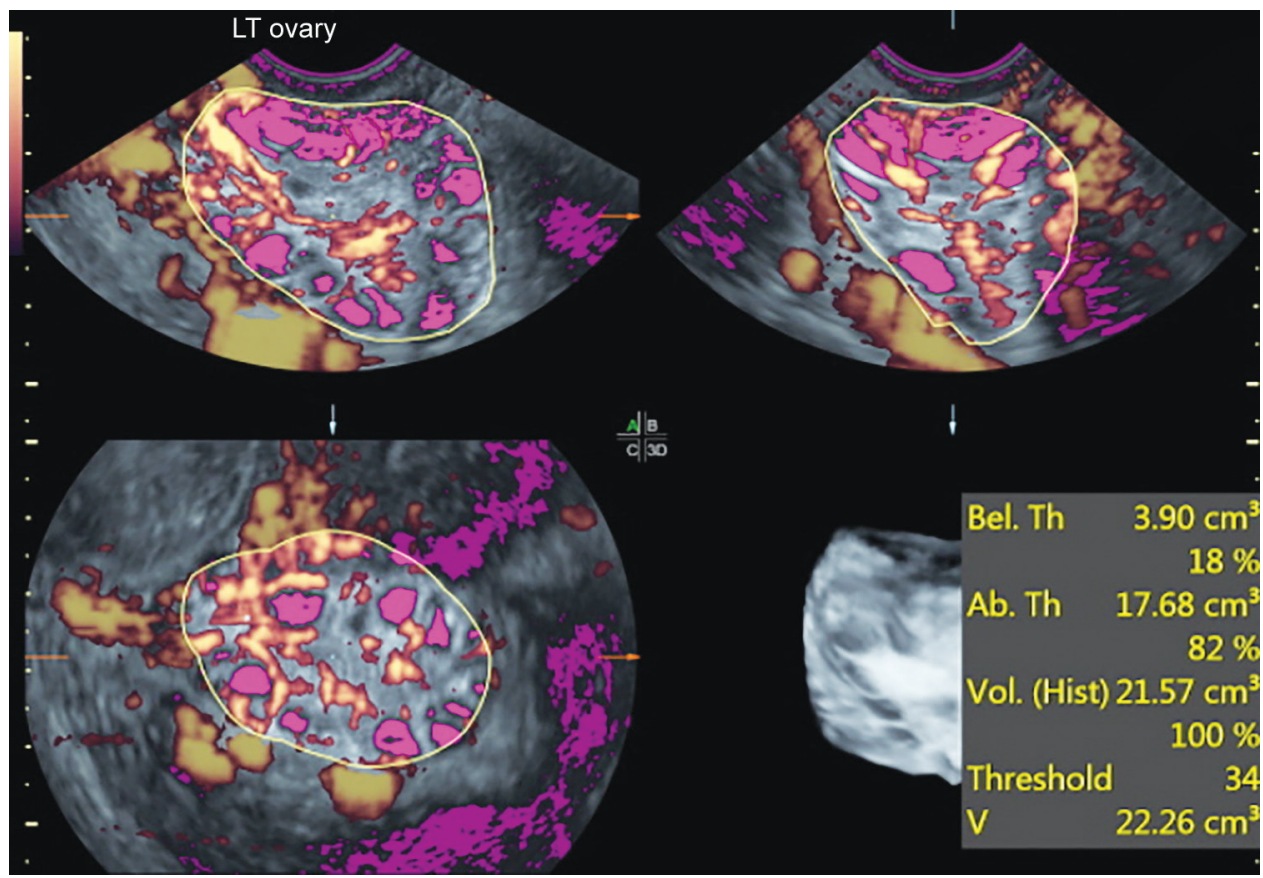

Fig. 38: 3D ultrasound acquired, VOCAL calculated ovarian volume with threshold volume used to calculate stromal volume of the ovary

frequent and specific feature in ovarian androgenic dysfunction. ${ }^{31}$ Polycystic ovarian morphology (PCOM) (stromal echogenicity) in normal women are not a morphological variant of normal ovaries but rather represent a functional entity-a silent form of PCOS. ${ }^{32}$ Stromal abundance and echogenicity is one of the major features that discriminate PCO from multicystic ovary (MCO). This can be assessed stromal echogenicity, stromal area, and stromal volume. Polycystic ovaries show a hyperechoic ${ }^{33}$ stroma and by ovarian area: $5.5 \mathrm{~cm}^{2}$ (93\% sensitivity and $91 \%$ specificity)—strict longitudinal ovarian section and by stromal area: $4.6 \mathrm{~cm}$ seconds ${ }^{2}$ ( $91 \%$ sensitivity and $86 \%$ specificity) ${ }^{34}$ But the most accurate assessment of stromal abundance can only be with ovarian and stromal volume by 3D US. Three-dimensional US has the potential to address these points and improve the sensitivity and specificity of US in the diagnosis of PCOS. ${ }^{35}$

Both total ovarian volume and stromal volume during the early follicular phase are significantly higher in women with $\mathrm{PCOS}^{36}$ Stromal volume was positively correlated with serum androstenedione concentrations in patients with the polycystic ovarian syndrome. ${ }^{36}$ Statistically significant relationship was reported between ovarian volume and stroma echogenicity with serum luteinizing hormone (LH) and testosterone concentrations. ${ }^{37}$ Ovarian volume can be calculated by VOCAL as mentioned earlier and applying threshold volume to this can calculate the stromal volume. After the volume is calculated by VOCAL when threshold volume is activated, pigment appears on the VOCAL calculated volume. The threshold is so adjusted that the pigmented area fills up all the follicles and only follicles. On the screen above the threshold and below threshold volumes are displayed. Below the threshold is the follicular volume and above threshold is the stromal volume (Fig. 38). In PCOS patients, a strong and similar correlation is seen between ovarian and stromal volumes to fasting and postprandial insulin levels. ${ }^{38}$

Three-dimensional US not only permits improved spatial awareness and volumetric and quantitative vascular assessment but also provides a more objective tool to examine stromal echogenicity through the assessment of the mean greyness of the ovary (Fig. 39). ${ }^{39}$ Stromal index (stromal echogenicity/total ovarian echogenicity was significantly higher in PCOS than controls. ${ }^{30}$

Moreover, 3D US has clearly shown higher AFC (median 16.3 vs 5.5 per ovary), ovarian volume ( 12.56 vs $5.6 \mathrm{~mL}$ ), stromal volume (10.79 vs $4.69 \mathrm{~mL}$ ), and stromal vascularization (vascularization index (VI) 3.85 vs $2.79 \%$, vascularization flow index (VFI) 1.27 vs 0.85 ) in PCO patients. ${ }^{40}$ Ovarian stromal flow index (FI) is higher (33.94 vs 29.30) in hirsute than in normoandrogenic PCOS women. But in PCOS women with obesity, the vascularity was lower than in normal-weight women (VI 3.25 vs $4.51 \%$, VFI 1.22 vs 1.56 ). ${ }^{40}$ It is this vascularity that is responsible for hyperstimulation in patients with PCOS.

To summarize the morphological features of PCO that correlate the most with the biochemical derangements of PCOS, ovarian volume, stromal volume, and AFC can all be better and more precisely assessed by and correlated with by 3D US.

\section{Follicular Monitoring}

- Assessment of ovarian reserve and response.

- Assessment of follicular maturity and endometrial receptivity.

\section{Assessment of Ovarian Reserve and Response}

Three-dimensional US is also being used to predict the ovarian response in patients on assisted reproductive techniques.

Predictors of ovarian response are enumerated as: ${ }^{41}$

- The number of antral follicles.

- Stromal flow: stromal FI.

- Total ovarian stromal area.

- Total ovarian volume.

Antral follicle count can be used reliably for assessment of ovarian response. The more exact value of AFC can be acquired by 3D US. Number of follicles $>12 \mathrm{~mm}$ on day of oocyte retrieval correlated significantly with AFC counted by 3D US rather than 2D US. ${ }^{27}$ 


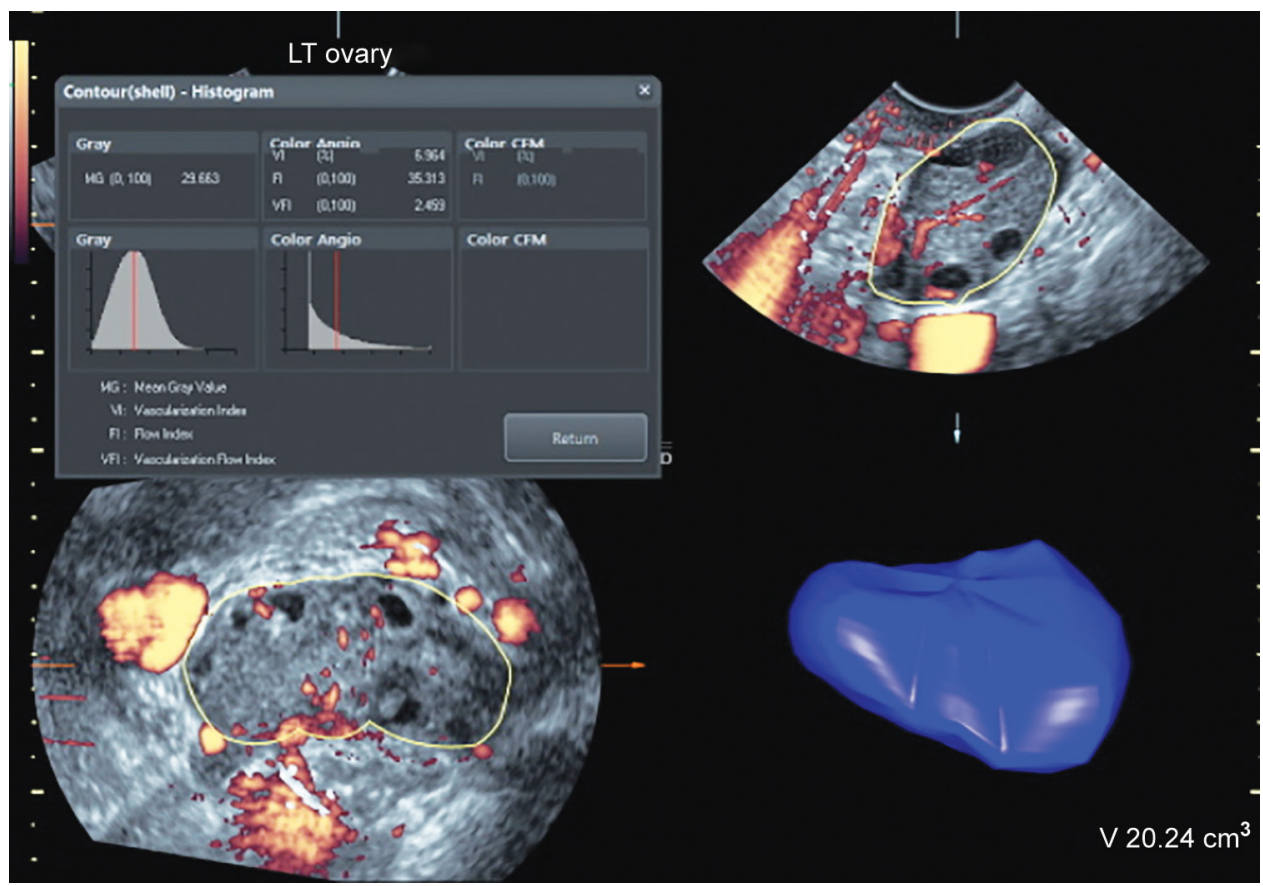

Fig. 39: 3D ultrasound acquired, VOCAL calculated ovarian volume with volume histogram, showing VI, FI and VFI values of the ovary

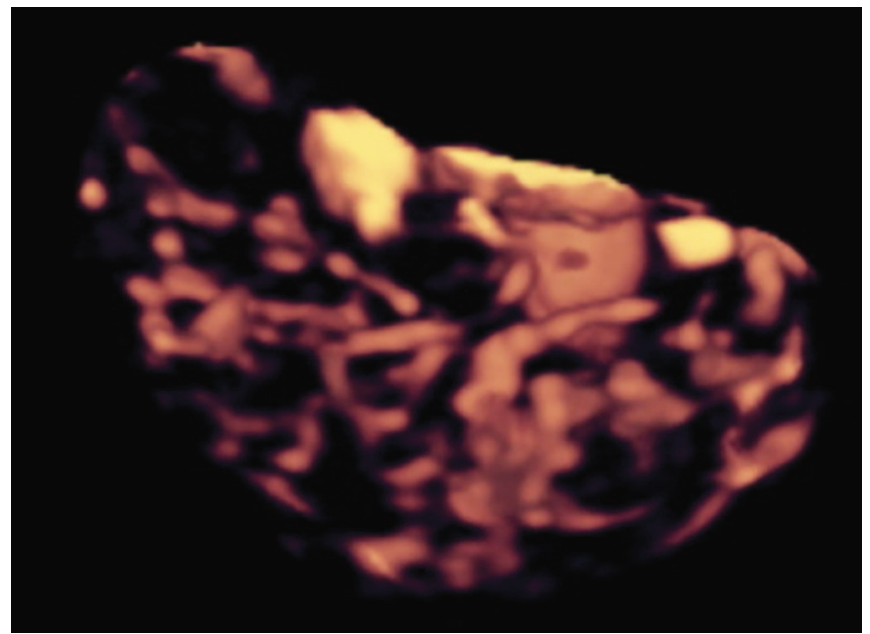

Fig. 40: 3D ultrasound acquired, VOCAL calculated ovarian volume rendered in 3D power Doppler angio mode showing ovarian global vascularity

Measurement of ovarian stromal flow in early follicular phase is related to subsequent ovarian response in IVF treatment ${ }^{42}$ (Fig. 40). Three-dimensional power Doppler with histogram can also be used to assess stromal vascularity on baseline scan. Kupesic et al, have shown a correlation in the ovarian stromal flow index and the number of mature oocytes retrieved in IVF cycles and pregnancy rates. Stromal FI ( $<11$ low responders, $11-14$ good, $>15$ risk of $\mathrm{OHSS}) .{ }^{41}$ Another group has demonstrated that VI, $\mathrm{FI}$, and VFI of the ovary were significantly related to ovarian response to stimulation. ${ }^{43}$

\section{Pretrigger Evaluation of Follicle and Endometrium Assessment of Follicular Maturity}

A follicle that is $16-18 \mathrm{~mm}$ in diameter and shows perifollicular vascularity covering 3/4th of the circumference of the follicle with
$\mathrm{RI}$ of $\leq 0.48$ and peak systolic velocity (PSV) of $10 \mathrm{~cm} / \mathrm{second}$ or more it is known to be functionally mature. Three-dimensional US and 3D power Doppler has been used for further assessment of these follicles.

On 3D the follicular volume of 3-7.5 cc has been found to be optimum in our study ${ }^{5}$ (Fig. 41). ${ }^{44}$ Follicular volumes of between 3 cc and $7 \mathrm{cc}$ are optimum for oocyte retrieval by VOCAL. The limits of agreement between the volume of the follicular aspirate and $3 \mathrm{D}$ volume of the follicle were +0.96 to -0.43 with $3 \mathrm{D}$ and +3.47 to -2.42 by $2 \mathrm{D}$ volume estimation. ${ }^{45}$

This is because 3D US measurement is not affected by the follicular shape as the changing contours are outlined serially to obtain the specific volume measurement. Though the follicles of $<10 \mathrm{~mm}$ in diameter, cannot be assessed accurately by 3D US because limits of agreement are too wide in this range.

After the volume calculation, the follicle is seen plane by plane in the acquired volume by translation and rotation that is walking through the volume and rotating the volume. Using the surface smooth or light gradient mode for rendering with a high threshold can show beautiful cumulus (Fig. 9). Feichtinger et al. in their study have shown a presence of cumulus in follicles $>15 \mathrm{~mm}$ by 3D US (Fig. 42). ${ }^{46}$ Follicles without visualization of cumulus in all three planes are not likely to contain mature oocytes. Poehl et al. ${ }^{9}$ also found a significant correlation between the number of detected cumuli by the 3D US and the number of retrieved oocytes $(P<0.0001)$, mature oocytes $(P<0.0001)$, and number of fertilized oocytes $(P<0.0001) .{ }^{47}$

It has also been suggested that the follicles containing oocytes capable to produce a pregnancy have a perifollicular vascular network more uniform and distinctive. ${ }^{48}$ Although it is possible to assess the follicular flow as expressed by the peak systolic velocity and perifollicular color map. ${ }^{41}$ it is the 3D power Doppler which proves the most precise information about the vascularization and follicular blood flow. Three-dimensional power Doppler gives a global assessment of the vascularity, or follicular perfusion, both qualitatively and quantitatively. 


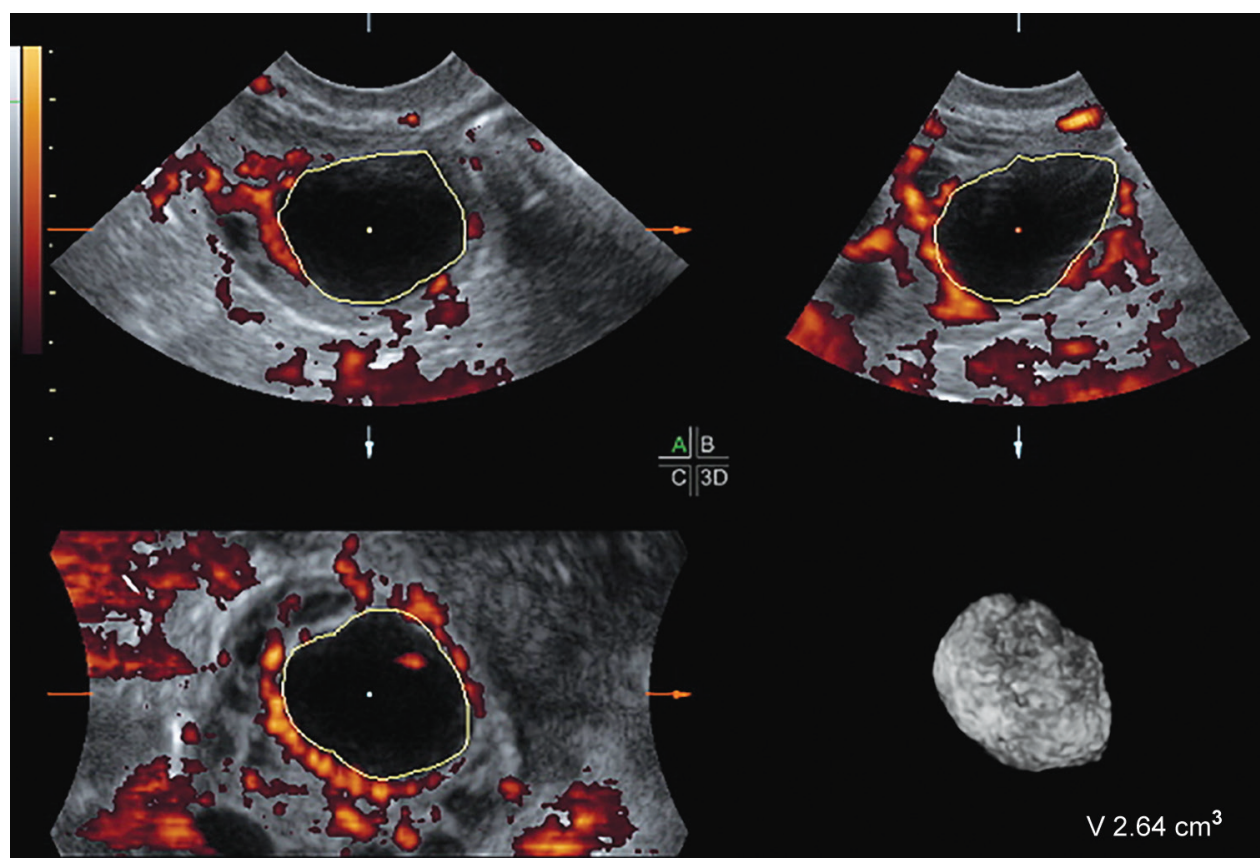

Fig. 41: 3D ultrasound acquired, VOCAL calculated follicular volume

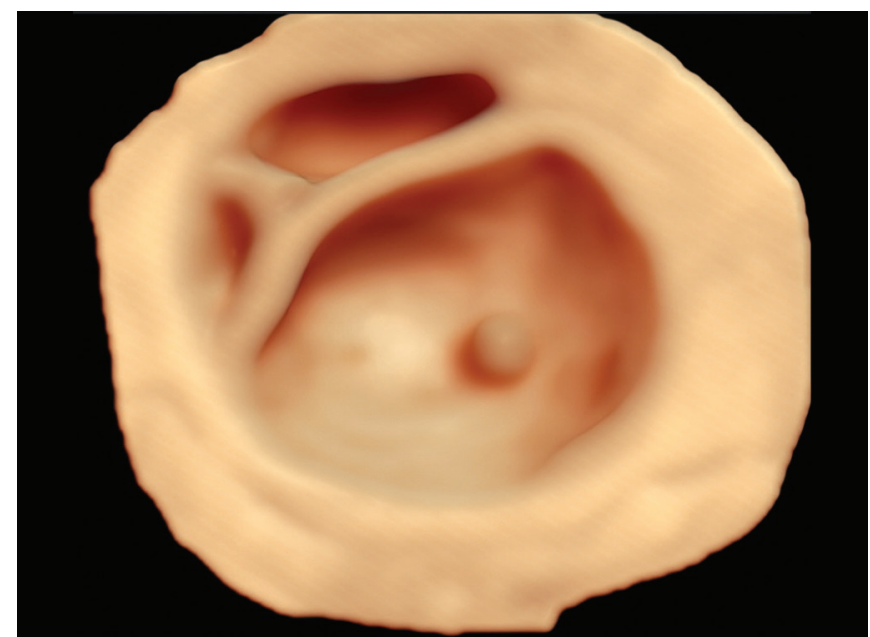

Fig. 42: 3D ultrasound acquired follicular volume and rendered on HD live mode, showing the cumulus

The pulse repetition frequency (PRF) settings for 3D power Doppler are fixed at 0.3 always and a 3D volume with power Doppler is taken. For the assessment of the perifollicular vascularity, the vascularity is assessed in the outside shell with the wall thickness of $2 \mathrm{~mm}$, which has been found to be the most appropriate to include the perifollicular vessels (Fig. 43A). Volume histogram of this shell will give $\mathrm{VI}, \mathrm{Fl}$, and VFI values of the perifollicular vessels (Fig. 43B).

We, in our study, have found perifollicular VI of between 6 and 20 and perifollicular $\mathrm{FI}>35$ as most optimum for fertilizable oocyte. About $68.4 \%$ of patients conceived when VI was between 6 and 18, and $50 \%$ when it was between 18 and 20 . However, the pregnancy rates were $<25 \%$ when $\mathrm{VI}$ was $<6$ and only $7.4 \%$ when VI was $>20 .{ }^{44}$

Meaning that even when the follicle appeared mature according to the 2D US and color and pulse Doppler parameters, the pregnancy rates were significantly better only when the follicular volume was between 3 and $7.5 \mathrm{cc}$, cumulus was present and the perifollicular VI was between 6 and 20 and $\mathrm{FI}$ was $>27$.

A study by Kupesic and Kurjak shows that when the ratio of follicular volume to blood flow index (FV/FI) is between 0.4 and 0.6 the pregnancy rates are $39 \%$, if $>0.6$, it is $52 \%$ and when $<0.4$ is only $21 \%{ }^{41}$

\section{Assessing Endometrial Receptivity}

Endometrium, like follicle, is assessed by transvaginal 2D US and color Doppler. Endometrial thickness of minimum $6 \mathrm{~mm}$ is required on the day of trigger, but $8 \mathrm{~mm}$ is optimum with a multilayered morphology, preferably grade $A$ or $\mathrm{B}^{49}$ (Fig. 44).

Segmental uterine artery perfusion demonstrates a significant correlation with hormonal and histological markers of uterine receptivity, reaching the highest sensitivity for subendometrial blood flow. ${ }^{50}$ On color Doppler the endometrium which is mature shows vascularity in zone 3 and 4 or maybe said in subendometrial and endometrial layers ${ }^{51}$ (Fig. 45). Zaidi et al. found that the absence of flow in the endometrial and subendometrial zones on the day of hCG indicates total failure of implantation. ${ }^{19}$ The vessels that reach the endometrium are the spiral arteries. ${ }^{52}$

Our study ${ }^{36}$ showed that at the endometrial volume of $<2$ cc no pregnancies occurred. With the endometrial volume of $2-3$ cc only $16.66 \%$ of patients conceived, between $3 c c$ and 5 cc $47 \%$ and when the endometrial volume was between $5 \mathrm{cc}$ and $7 \mathrm{cc} 61.5 \%$ patients conceived. ${ }^{45}$ Endometrial volume by 3D US volume calculation of the endometrium may help to correlate the cycle outcome with quantitative parameter rather than endometrial thickness ${ }^{52}$ (Fig. 46). For endometrial volume, the inter CC definition of internal os and (interobserver variation) was 0.82 and intra-CC (intraobserver variation) was 0.90 , the chief source of error being a definition of endometrial margins. ${ }^{45}$

A study by Raga et al. ${ }^{53}$ shows pregnancy and implantation rates were significantly lower when endometrial volume $<2 \mathrm{~mL}$, 

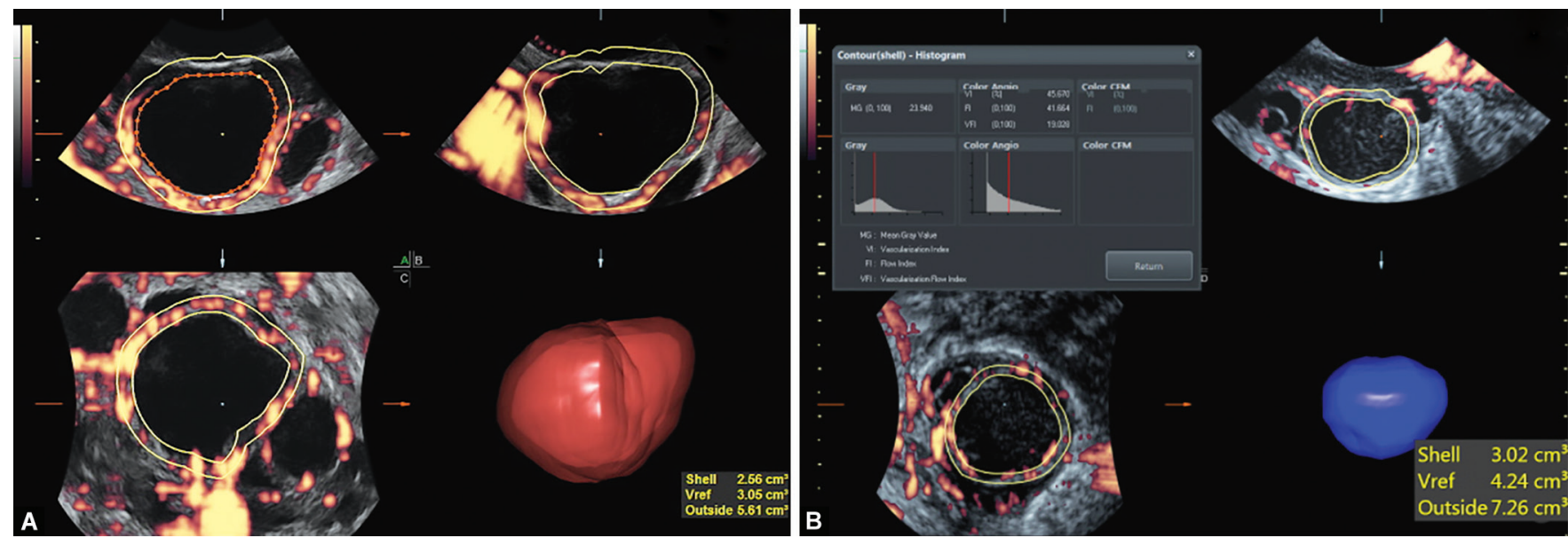

Figs 43A and B: (A) 3D power Doppler ultrasound acquired follicular volume, with VOCAL calculated volume of the follicle with shell of $2 \mathrm{~mm}$; (B) Volume histogram applied on the shell volume of follicle showing $\mathrm{VI}, \mathrm{FI}$, and $\mathrm{VFI}$ values of the perifollicular flow
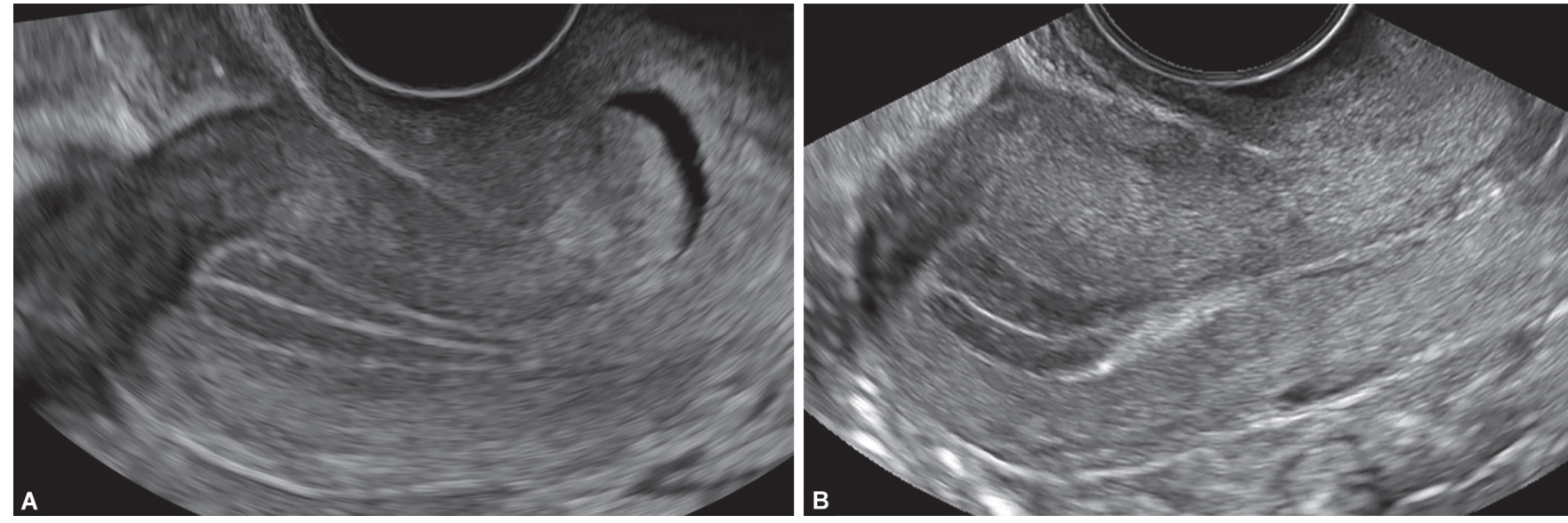

Figs 44A and B: B mode ultrasound image of the uterus showing grade A and grade B endometrium, respectively

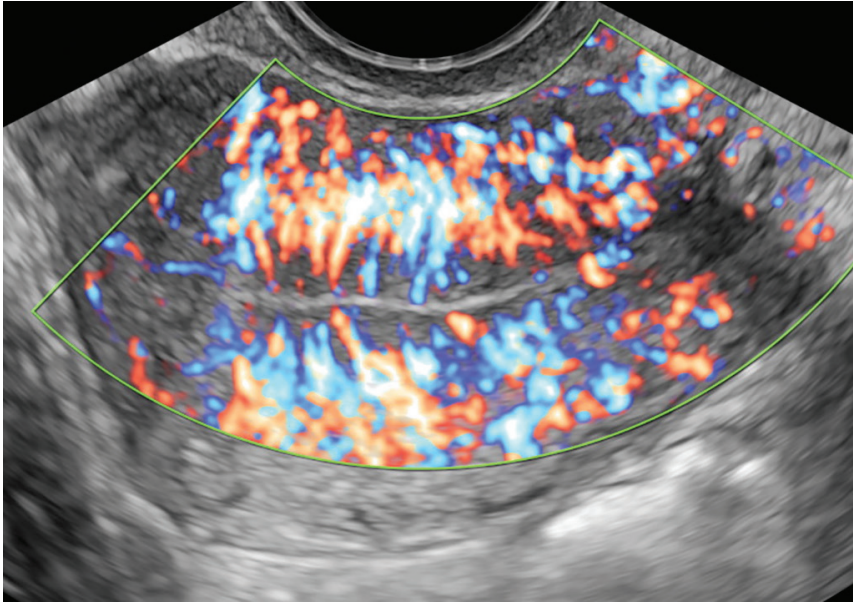

Fig. 45: HD flow image showing zone 3, 4 vascularity of the endometrium

while no pregnancy was achieved when the endometrial volume was $<1 \mathrm{~mL}$. A study by Kupesic et al. also shows no pregnancy when the endometrial volume was $<2 \mathrm{~mL}$, or when exceeded $8 \mathrm{~mL}$. ${ }^{54}$ For the same calculated volume, volume histogram is switched on and it calculates the endometrial VI, Fl, and VFI (Fig. 47).

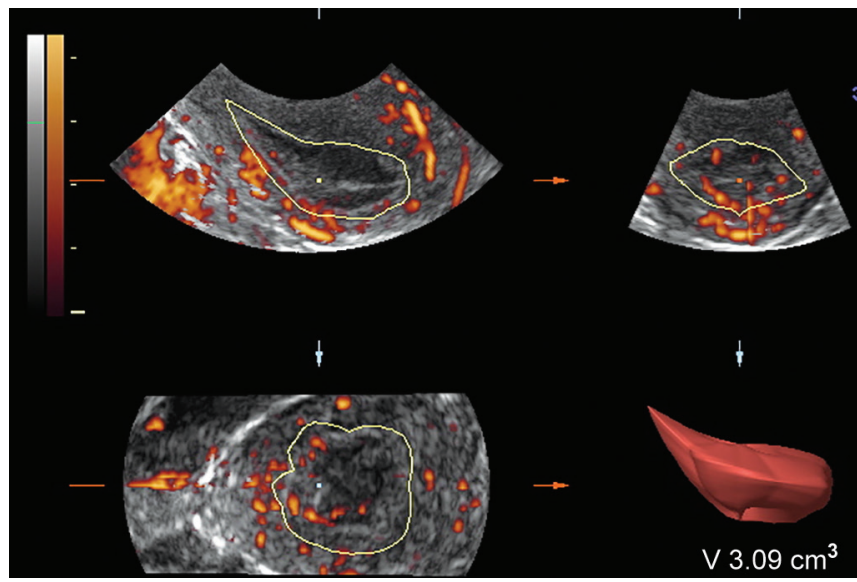

Fig. 46: 3D power Doppler acquired, VOCAL calculated volume of the endometrium

A scoring system reported by Kupesic et al., ${ }^{24}$ for uterine receptivity, done on the day of embryo transfer shows that subendometrial $\mathrm{FI}<11$ was a cut-off limit. (Subendometrial indices are calculated in the same way from the endometrial volume as we calculate the perifollicular indices from the follicular volume, that 


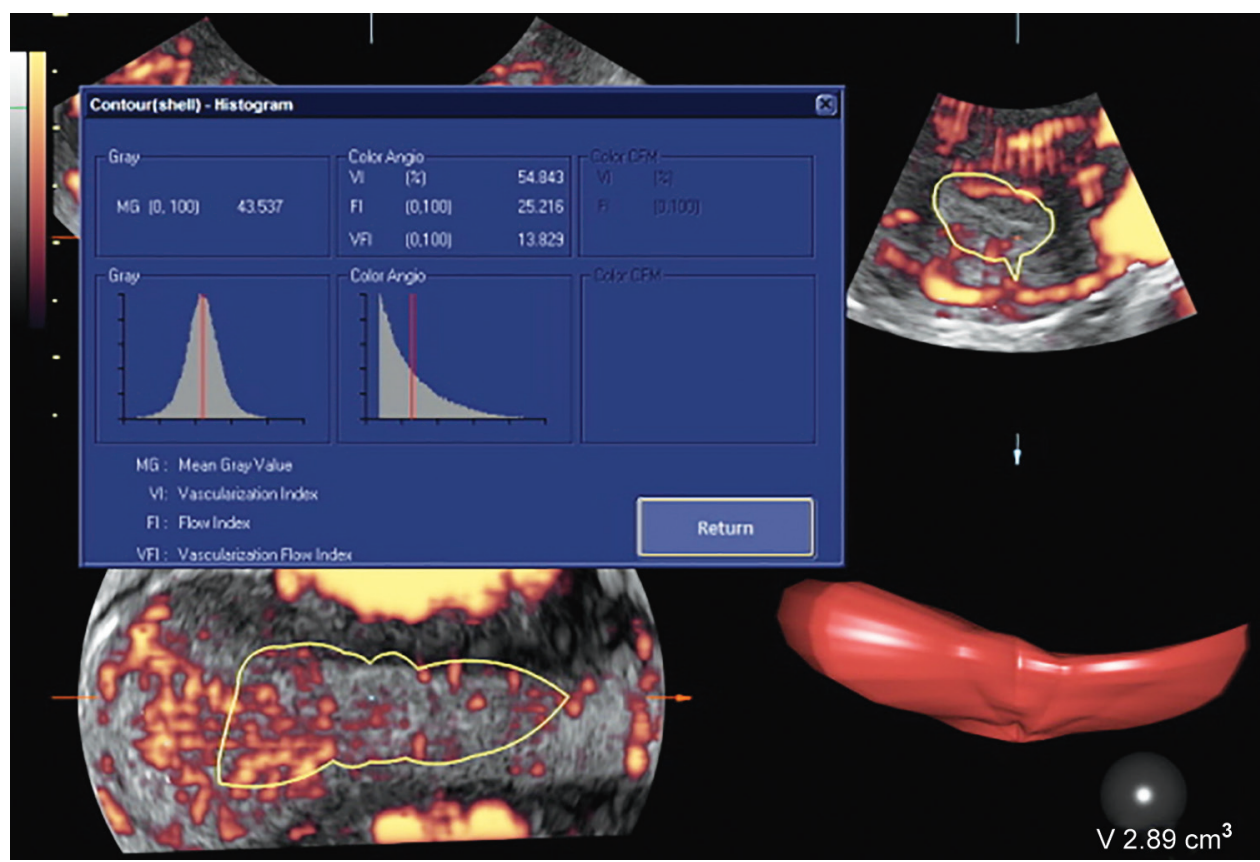

Fig. 47: 3D power Doppler acquired, VOCAL calculated volume of the endometrium with volume histogram showing global vascularity indices $\mathrm{VI}, \mathrm{Fl}$, and $\mathrm{VFI}$ values of the endometrium
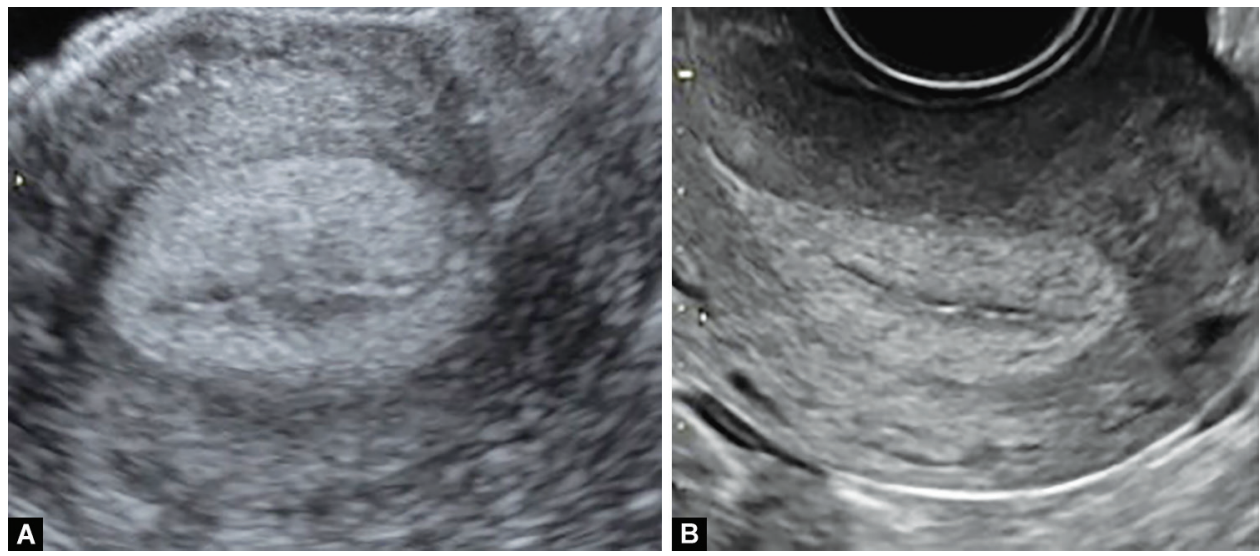

Figs 48A and B: (A) B mode ultrasound image of the endometrium showing asymmetrical thickness of the endometrial lips; (B) B mode ultrasound image of the endometrium showing symmetrical thickness of the endometrial lips

is, by shell volume of $2 \mathrm{~mm}$ thickness). No pregnancies occurred when it was $<11$ and the conception group showed its values of $13.2 \pm 2.2^{54}$

Contrary to this $\mathrm{Ng}$ et al. documented a low endometrial VI and VFI in a pregnant group on the day of oocyte retrieval and also a non-significant trend of higher implantation and pregnancy rates in patients with absent subendometrial and endometrial flow. ${ }^{55}$ Though they concluded that the number of embryos replaced and the endometrial VFI were the only two predictive factors for pregnancy. Wu et al. ${ }^{56}$ reported that endometrial VFI was more reliable than $\mathrm{VI}$ and $\mathrm{Fl}$, and the best prediction rate was achieved by VFI cut off value of $>0.24$.

Collectively the evidence from various studies suggests that adding 3D and 3D power Doppler for the assessment of follicular maturity and endometrial receptivity, improves the outcome of the fertility treatment by assessment of global vascularity.

\section{Ectopic Pregnancy}

The gestational sac that has implanted at places other from the upper body of the endometrial cavity. It can be in the fallopian tube (95\%), abdominal cavity, ovary, broad ligament, uterine cornu, myometrium, cervix, or scar. It is suspected when in a female with a missed period and beta hCG level of $>1500 \mathrm{IU} / \mathrm{mL}$, on US does not show an intrauterine gestational sac. In case of an early intrauterine pregnancy, the endometrial lips are asymmetrical (Fig. 48A) and in the case of ectopic pregnancy, the lips of endometrium are symmetrical (Fig. 48B). This may be easily appreciated also on $B$ mode US. The true gestational sac is eccentric in the sac and shows peripheral vascularity. Any adnexal lesion in such a female that is tender on probe pressure or shows peripheral ring of vascularity is an ectopic (Fig. 49). Tubal pregnancies can be diagnosed by $B$ mode and color Doppler. It is the differentiation between interstitial pregnancy and angular pregnancy, diagnosis 

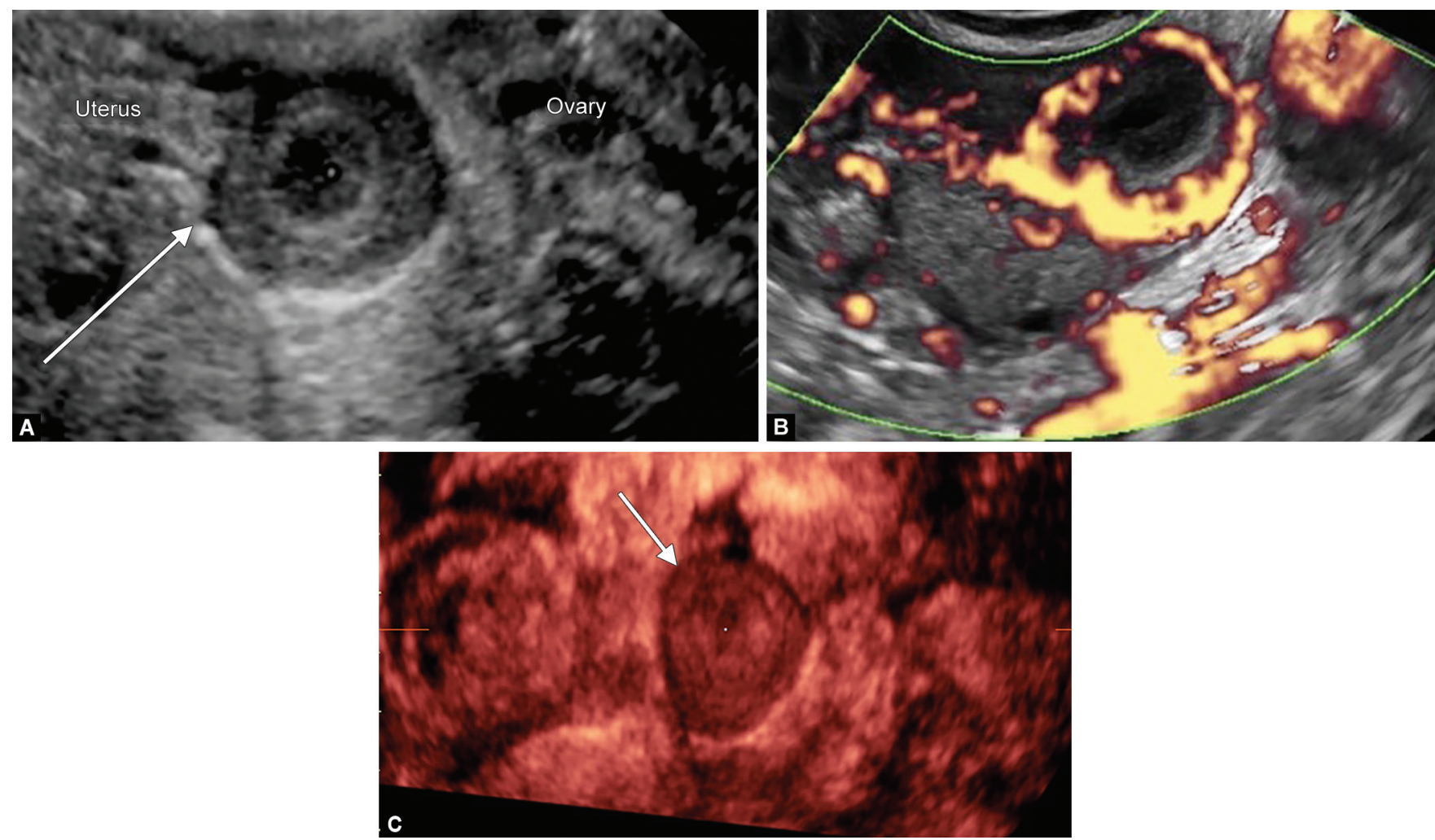

Figs 49A to C: (A) B mode ultrasound image of the adnexa showing a round isoechoic mass with central anechoic area with hyperechoic rim suggesting an ectopic pregnancy; (B) On power Doppler the lesion shows a ring of color-peripheral vascularity; (C) 3D Ultrasound showing coronal plane of the adnexal with ectopic pregnancy mass
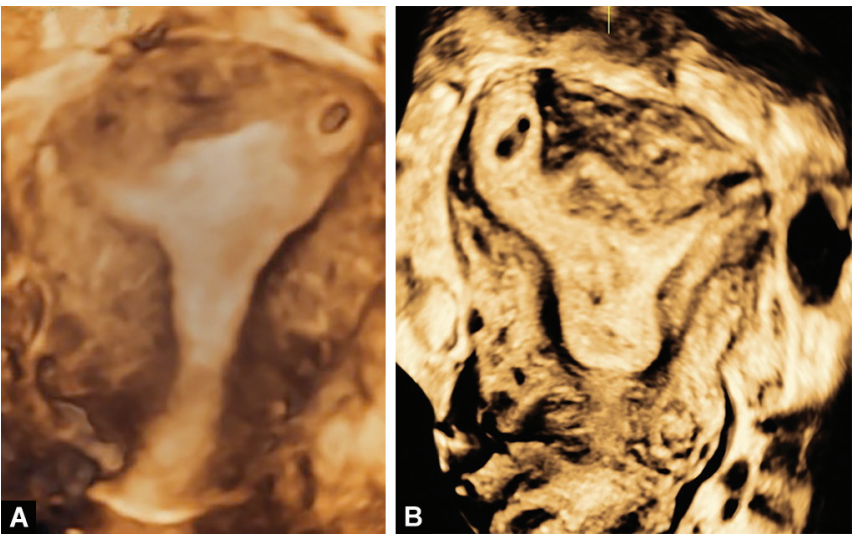

Figs 50A and B: (A) 3D rendered image of the uterus showing left angular pregnancy; $(B)$ 3D rendered image of the uterus showing interstitial pregnancy

of cervical pregnancy, and scar pregnancy, for which 3D plays an important role. Interstitial pregnancy on a coronal plane of the uterus clearly shows a myometrial gap between the gestational sac and the endometrial cavity, unlike the angular pregnancy in which the endometrium covers one side of the gestational sac but not the other side (Fig. 50). Cervical pregnancy typically leads to expansion of the cervix due to progressing pregnancy and leads to hourglass uterus, with gestational sac placed eccentrically in the cervix (Fig. 51). Scar pregnancies may be in the sac or over the scar and the prognosis for both is significantly different. Scar over

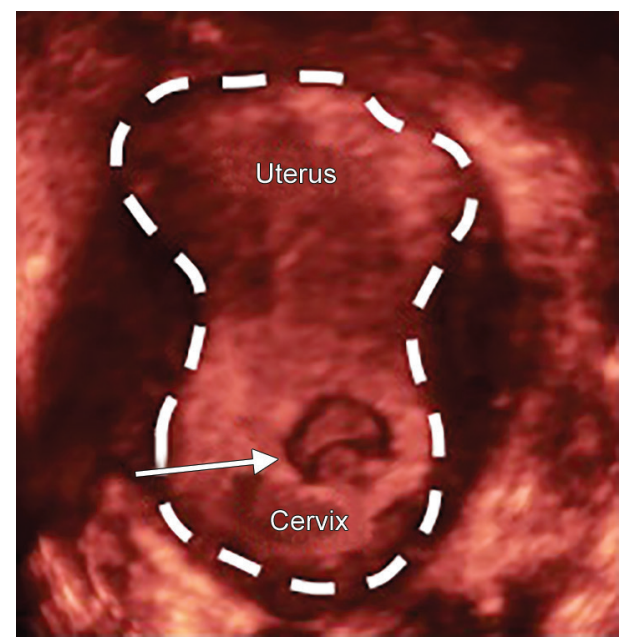

Fig. 51: 3D rendered image of the uterus showing cervical pregnancy and hourglass uterus

the pregnancy leads to the growth of the pregnancy in the uterus and the risk is the only adherent placenta. But for the pregnancy in the scar, the risk of rupture of the uterus is significantly high and requires immediate intervention (Fig. 52).

Apart from this even when conservative or interventional treatment is planned, with the onset of the medical line of treatment there is first decrease in the vascularity and that 3D and 3D PD will be a useful tool to diagnose that the regression of the lesion even before the size of the lesion decreases. Reduction in $\mathrm{VI}, \mathrm{FI}$, and VFI are useful to assess quantitative, (Fig. 53). 

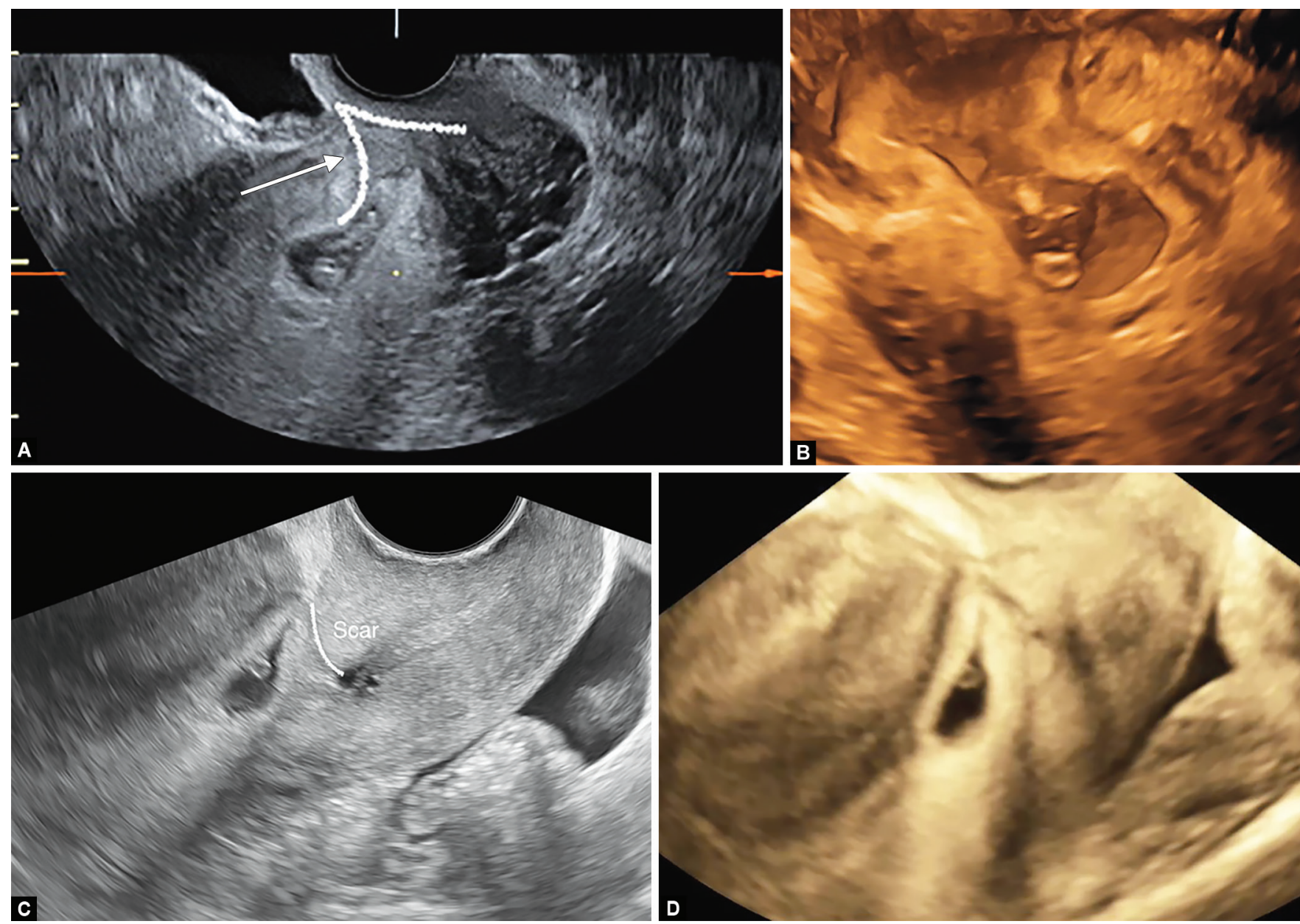

Figs 52A to D: (A) Pregnancy in the scar on B mode; (B) Pregnancy in the scar on 3D ultrasound; (C) B mode ultrasound image showing gestational sac over the scar; (D) 3D ultrasound with volume contrast imaging A confirming the diagnosis of pregnancy in the scar

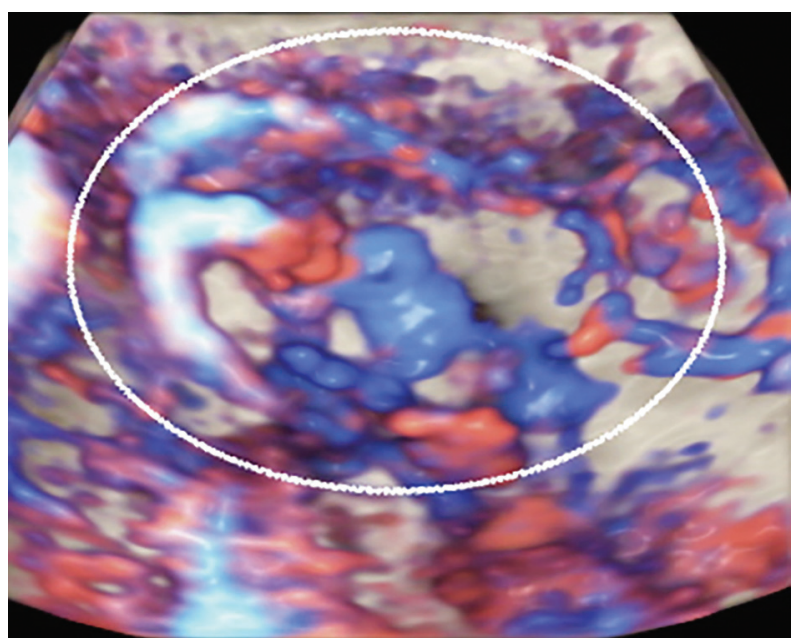

Fig. 53: 3D HD flow volume showing global vascularity of the ectopic pregnancy

\section{CONCLUSION}

Three dimensional US can better demonstrate the uterine lesions like polyps, endometrial synechiae and fibroids. Better delineation of endometrio-myometrial junction helps diagnosis of adenomyosis, comparable to MRI. Tubal assessment can be made more effective by adding 3D to HyCOSy. Three-dimensional US is accurate for volume assessment both for follicle and the endometrium, that are much more reliable parameters than the follicular diameter or endometrial thickness. The presence of cumulus, the presence of which can be confirmed by 3D US more easily than with $B$ mode and increases the surety of the presence of a mature ovum in the follicle. Three-dimensional power Doppler gives an idea about the global vascularity of the follicle and the endometrium. Though still larger studies are needed to establish more precise values for follicular and endometrial $\mathrm{VI}, \mathrm{FI}$, and $\mathrm{VFI}$, the results are fairly promising.

\section{References}

1. Cil AP, Tulunay G, Kose MF, et al. Power Doppler properties of endometrial polyps and submucosal fibroids: preliminary observational study in women with known intracavitary lesions. Ultrasound Obstet Gynecol 2010;35(2):233-237. DOI: 10.1002/ uog.7470.

2. de Souza NM, Brosens JJ, Schwieso JE, et al. The potential value of magnetic resonance imaging in infertility. Clin Radiol 1995;50(2): 75-79. DOI: 10.1016/S0009-9260(05)82983-6.

3. Exacoustos C, Brienza L, Di Giovanni A, et al. Adenomyosis: threedimensional sonographic findings of the junctional zone and correlation with histology. Ultrasound Obstet Gynecol 2011;37(4): 471-479. DOI: 10.1002/uog.8900. 
4. Van Den Bosch T, Dueholm M, Leone FP, et al. Terms, definitions and measurements to describe sonographic features of myometrium and uterine masses: a consensus opinion from the Morphological Uterus Sonographic Assessment (MUSA) group. Ultrasound Obstet Gynecol 2015;46(3):284-298. DOI: 10.1002/uog.14806.

5. Mavrelos D, Naftalin J, Hoo W, et al. Preoperative assessment of submucous fibroids by 3D saline contrast sonohysterography. Ultrasound Obstet Gynecol 2011;38(3):350-354. DOI: 10.1002/ uog.9049.

6. Crade M. Tissue block ultrasound and ovarian cancer-a pictorial presentation of findings. Ultrasound Obstet Gynecol 2009;3(1):41-47. DOI: 10.5005/jp-journals-10009-1007.

7. Raine-Fenning N, Jayaprakasan K, Deb S. Three dimensional ultrasonographic characteristics of endometriomata. Ultrasound Obstet Gynecol 2008;31(6):718-724. DOI: 10.1002/uog.5380.

8. Patel MD, Feldstein VA, Chen DC, et al. Endometriomas: diagnostic performance of US-NCBI. Radiology 1999;210(3):739-745. DOI: 10.1148/radiology.210.3.r99fe61739.

9. Van Holsbeke C, Zhang J, Van Belles V, et al. Acoustic streaming can not discriminate relaibly between endometriomas and other types of adnexal lesion: a multicenter study of 633 adnexal masses. Ultrasound Obstet Gynecol 2010;35(3):349-353. DOI: 10.1002/uog.7537.

10. Kurjak A, Kupesic S, Sparac V, et al. The detection of stage 1 ovarian cancer by three dimensional sonography and power Doppler. Gynecol Oncol 2003;90(2):258-264. DOI: 10.1016/S0090-8258(03)00205-1.

11. Kupesic S, Kurjak A. Contrast enhanced three dimensional power Doppler sonography for the differentiation of adnexal masses. Obstet Gynecol 2000;96(3):452-458. DOI: 10.1016/S0029-7844(00)00923-6.

12. Jeanty $P$, Besnard S, Arnold A, et al. Air-contrast sonohysterography as a first step assessment of tubal patency. J Ultrasound Med 2000;19(8):519-527. DOI: 10.7863/jum.2000.19.8.519.

13. HolzK, Becker R, Schürmann R. Ultrasound in the investigation of tubal patency: a meta-analysis of three comparative studies of Echovist-200 including 1007 women. Zentralbl Gynakol 1997;119(8):366-373.

14. Exacoustos C, Zupi E, Carusotti C, et al. Hysterosalpingo-contrast sonography compared with hysterosalpingography and laparoscopic dye pertubation to evaluate tubal patency. J Am Assoc Gynecol Laparosc 2003;10(3):367-372. DOI: 10.1016/S1074-3804(05)60264-2.

15. Balen FG, Allen CM, Gardener JE, et al. 3-Dimensional reconstruction of ultrasound images of the uterine cavity. British journal of Radiology 1993;66(787):588-591. DOI: 10.1259/0007-1285-66-787-588.

16. Exacoustos C, Di Giovanni A, Szabolcs B, et al. Automated threedimensional coded contrast imaging hysterosalpingo-contrast sonography: feasibility in office tubal patency testing. Ultrasound Obstet Gynecol 2013;41(3):328-335. DOI: 10.1002/uog.11200.

17. Chan CC, Ng EH, Tang OS, et al. Comparison of three-dimensional hysteron-contrast-sonography and diagnostic laparoscopy with chromopertubation in the assessment of tubal patency for the investigation of subfertility. Acta Obstet Gynecol Scand 2005;84(9):909-913. DOI: 10.1111/j.0001-6349.2005.00797.x.

18. Kupesic S, Plavsic MB. 2D and 3D hysterosalpingo contrastsonography in the assessment of uterine cavity and tubal patency. Eur J Obstet Gynecol Reprod Biol 2007;133(1):64-69. DOI: 10.1016/ j.ejogrb.2006.10.010.

19. Zhou L, Zhang X, Chen X, et al. Value of three -dimensional hysterosalpingo- contrast sonography with SonoVue in the assessment of tubal patency. Ultrasound Obstet Gynecol 2012;40(1):93-98. DOI: 10.1002/uog.11085.

20. Sladkevicius P, Ojha K, Campbell S, et al. Three-dimensional power Doppler imaging in the assessment of Fallopian tube patency. Ultrasound Obstet Gynecol 2000;16(7):644-647. DOI: 10.1046/j.14690705.2000.00302.x.

21. Lam PM, Raine-Fenning N. The role of three-dimensional ultrasonography in polycystic ovary syndrome. Hum Reprod 2006;21(9):2209-2215. DOI: 10.1093/humrep/del161.

22. Kupesic S, et al. Sonographic imaging in infertility. Donaldd School textbook of transvaginal Ultrasound, 1st edn, Jaypee Brothers Medical Publishers(P) Ltd; 2005. pp. 357-383.
23. Jonard S, Robert Y, Dewailly D. Revisiting the ovarian volume as a diagnostic criterion for polycystic ovaries. Hum Reprod 2005;20(10):2893-2898. DOI: 10.1093/humrep/dei159.

24. Legro RS, Chiu P, Kunselman AR, et al. Polycystic ovaries are common in women with hyperandrogenic chronic anovulation but do not predict metabolic or reproductive genotype. JCEM 90(5):2571-2579. DOI: 10.1210/jc.2004-0219.

25. Jonard S, Robert Y, Cortet-Rudelli C, et al. Ultrasound examination of polycystic ovaries: is it worth counting the follicles? Hum Reprod 2003;18(3):598-603. DOI: 10.1093/humrep/deg115.

26. Lujan ME, Jarrett BY, Brooks ED, et al. Updated ultrasound criteria for polycystic ovary syndrome: reliable thresholds for elevated follicle population and ovarian volume. Hum Reprod 2013;28(5):1361-1368. DOI: 10.1093/humrep/det062.

27. $\mathrm{Ng} \mathrm{EH}, \mathrm{Chan} \mathrm{CC}$, Yeung WS, et al. Effect of age on ovarian stromal flow measured by three-dimensional ultrasound with power Doppler in Chinese women with proven fertility. Hum Reprod 2004;19(9): 2132-2137. DOI: 10.1093/humrep/deh387.

28. Deb S, Campbell BK, Clewes JS, et al. Quantitative analysis of AFC and size: a comparison of 2D \& automated three-dimensional ultrasound techniques. Ultrasound Obstet Gynecol 2010;35(3):354-360. DOI: 10.1002/uog.7505.

29. Defining PCOS-a perspective: Dr. Thatcher, The American Infertility Association News letter; February 2001.

30. Buckett WM, Bouzayeb R, Watkin KL, et al. Ovarian stromal echogenicity in women with normal and polycystic ovaries. Hum Reprod 1999;14(3):618-621. DOI: 10.1093/humrep/14.3.618.

31. Falghesu AM, Angioni S, Frau E, et al. Ultrasound in polycystic ovary syndrome- the measuring of ovarian stroma and relationship with circulating androgens: results of a multicentric study. Hum Reprod 2007;22(9):2501-2508. DOI: 10.1093/humrep/dem202.

32. Franks $\mathrm{S}$, Webber $L J$, Goh $M$, et al. Ovarian morphology is a marker of heritable biochemical traits in sisters with polycystic ovaries. J Clin Endocrinol Metab 2008;93(9):3396-3402. DOI: 10.1210/jc.2008-0369.

33. Robert Y, Dubrulle F, Gaillandre L, et al. Ultrasound assessment of ovarian stroma hypertrophy in hyperandrogenism and ovulation disorders: visual analysis versus computerized quantification. Fertil Steril 1995;64(2):307-312. DOI: 10.1016/S0015-0282(16)57728-0.

34. Dewailly $D$, Robert $Y$, Helin I, et al. Ovarian stromal hypertrophy in hyperandrogenic women. Clin Endocrinol 1994;41(5):557-562. DOI: 10.1111/j.1365-2265.1994.tb01818.x.

35. Raine-Fenning NJ,Campbell BK, Clewes JS, et al. The reliability of virtual organ computer-aided analysis (VOCAL) for the semiquantification of ovarian, endometrial and subendometrialperfusion. Ultrasound Obstet Gynecol 2003;22(6):633-639. DOI: 10.1002/uog.923.

36. Kyei-Mensah AA, LinTan S, Zaidi J, et al. Relationship of ovarian stromal volume to serum androgen concentrations in patients with polycystic ovary syndrome. Hum Reprod 1998;13(6):1437-1441. DOI: 10.1093/humrep/13.6.1437.

37. Pache TD, de Jong FH, Hop WC, et al. Association between ovarian changes assessed by transvaginal sonography and clinical and endocrine signs of the polycystic ovary syndrome. Feril Steril 1993;59(3):544-549. DOI: 10.1016/S0015-0282(16)55797-5.

38. Panchal S, Nagori CB. Assessing correlation between ovarian \& stromal volumes and fasting \& postprandial insulin levels in PCOS patients. Int J Infertil Fetal Med 2014;5(1):12-14. DOI: 10.5005/ jp-journals-10016-1073.

39. Jarvela IY, Mason HD, Sladkevicius $\mathrm{P}$, et al. Characterization of normal and polycystic ovaries using three dimensional power Doppler ultrasonography. J Assist Reprod Genet 2002;19(12):582-590. DOI: 10.1023/A:1021267200316.

40. Lam PM, Johnson IR, Rainne-Fenning NJ. Three dimensional ultrasound features of the polycystic ovary and the effect of different phenotypic expressions on these parameters. Hum Reprod 2007;22(12):3116-3123. DOI: 10.1093/humrep/dem218.

41. Kupesic S, Kurjak A. Predictors of IVF outcome by three dimensional ultrasound. Hum Reprod 2002;17(4):950-955. DOI: 10.1093/ humrep/17.4.950. 
42. Zaidi J, Barber J, Kyei-Mensah A, et al. Relationship of ovarian stromal blood flow at baseline ultrasound to subsequent follicular response in an in vitro fertilization program. Obstet Gynecol 1996;88(5):779-784. DOI: 10.1016/0029-7844(96)00316-X.

43. Merce LT, Barco MJ, Bau S, et al. Prediction of ovarian response and IVF/ICSI outcome by three-dimensional ultrasonography and power Doppler angiography. Eur J Obstet Gynecol Reprod Biol 2007;132(1):93-100. DOI: 10.1016/j.ejogrb.2006.07.051.

44. Panchal SY, Nagori CB. Can 3D PD be a better tool for assessing the pre HCG follicle and endometrium? A randomized study of 500 cases. Presented at 16th World Congress on Ultrasound in Obstetrics and Gynecology, 2006, London. J Ultrasound Obstet Gynecol 2006;28(4):504. DOI: 10.1002/uog.3361.

45. Kyei-Mensah A, Zaidi J, Pittrof $R$, et al. Transvaginal three dimensional ultrasound: accuracy of follicular volume measurements. Fertil Steril 1996;65(2):371-376. DOI: 10.1016/S0015-0282(16)58102-3.

46. Feichtinger $W$. Transvaginal three dimensional imaging for evaluation and treatment of infertility. In 3D Ultrasound in Obstetrics and Gynecology Merz E, ed. Philadelphia: Lippincott Williams and Wilkins; 1998. pp. 37-43.

47. Poehl M, Hohlagschwandtner M, Doerner V, et al. Cumulus assessment by three dimensional ultrasound for in vitro fertilization. Ultrasound Obstet Gynecol 2000;16(3):251-253. DOI: 10.1046/j.14690705.2000.00270.x.

48. Vlaisavljevic V, Reljic M, Gavric Lovrec V, et al. Measurement of perifollicular blood flow of the dominant preovulatory follicle using three dimensional power doppler. Ultrasound Obstet Gynecol 2003;22(5):520-526. DOI: 10.1002/uog.225.

49. Smith B, Porter R, Ahuja K, et al. Ultrasonic assessment of endometrial changes in stimulated cycles in an in vitro fertilization and embryo transfer program. J. In Vitro Fertil. Embryo Transf 1984;1(4):233-238. DOI: 10.1007/BF01131622.
50. Merce LT, Barco MJ, Kupesic S, et al. 2D and 3D power doppler ultrasound from ovulation to implantation. In Textbook of perinatal medicine Kurjak A, Chervenak F, ed. London: Parthenon Publishing; 2005.

51. Applebaum M. The 'steel' or 'teflon' endometrium - ultrasound visualization of endometrial vascularity in IVF patients and outcome. Presented at The third World Congress of Ultrasound in Obstetrics and Gyneacolgy. Ultrasound Obstet Gynecol 1993;3(Suppl 2):10.

52. Zaidi J, Campbell S, Pittrof R, et al. Endometrial thickness, morphology, vascular penetration and velocimetry in predicting implantation in an in vitro fertilization program. Ultrasound Obstet Gynecol 1995;6(3):191-198. DOI: 10.1046/j.1469-0705.1995. 06030191.x.

53. Raga F, Bonilla-Musoles F, Casan EM, et al. Assessment of endometrial volume by three dimensional ultrasound prior to embryo transfer: clues to endometrial receptivity. Hum Reprod 1999;14(11):2851-2854. DOI: 10.1093/humrep/14.11.2851.

54. Kupesic S, Bekavac I, Bjelos D, et al. Assessment of endometrial receptivity by transvaginal colour doppler and three dimensional power doppler ultrasonography in patients undergoing in vitro fertilization procedures. J Ultrasound Med 2001;20(2):125-134. DOI: 10.7863/jum.2001.20.2.125.

55. Ng EH, Chan CC, Tang OS, et al. The role of endometrial and subendometrial blood flows measured by three dimensional power doppler ultrasound in prediction of pregnancy during IVF treatment. Hum Reprod 2006;21(1):164-170. DOI: 10.1093/humrep/ dei277.

56. Wu HM, Chiang $\mathrm{CH}$, Huang $\mathrm{HY}$, et al. Detection of subendometrial vascularization flow index by three dimensional ultrasound may be useful for predicting pregnancy rate for patients undergoing in vitro fertilization-embryo transfer. Fertil Steril 2003;79(3):507-511. DOI: 10.1016/S0015-0282(02)04698-8. 